\title{
Virtual decoupling of vibroacoustical systems
}

\author{
Sifeng Bi ${ }^{1, *}$, Morvan Ouisse ${ }^{1}$, Emmanuel Foltête ${ }^{1}$, Arnaud Jund ${ }^{21}$ \\ ${ }^{1}$ Femto-ST Institute, Department of Applied Mechanics, University of Bourgogne Franche-Comté \\ 25000 Besancon, France \\ ${ }^{2}$ PSA Group, Research and Development Division, Technique Centre of Belchamp \\ 25218 Montbéliard Cedex, France
}

\begin{abstract}
Vibroacoustical systems as well as their behavior are coupled by nature. However, it may be of first interest for engineers to work on decoupled models, so that behavior of the structural/acoustic sub-system can be easily predicted and investigated. This work focuses on a virtual decoupling approach for vibroacoustics, with the objective to reconstruct decoupled and reduced system matrices from coupled experimental measurements. As a promising identification technique, the properness enforcement method has been developed in the literature for both symmetric and non-symmetric systems. During the decoupling process, however, this methodology still needs to be improved from two aspects: 1) the introduction of an additional correlation process so that the structural/acoustic sub-model can be correctly extracted from the coupled system; and 2) the additional optimization step after the complex vectors are approximately corrected by the properness enforcement method. These two key points are addressed by an integrated framework containing three aspects (i.e. identification, optimization, and correlation), which are specially designed for vibroacoustical applications. The finally identified system matrices of a decoupled and reduced equivalent system can exhibit the same behavior as the experimentally measured one. A simulated example is first presented to illustrate the use of this approach in detail. Then an experimental case study is used to demonstrate its feasibility in engineering applications.
\end{abstract}

Keywords: vibroacoustics; system identification; properness condition; matrix identification; experimental modal analysis; complex modes

\section{Introduction}

Decoupled and reduced models of the acoustic and structural parts extracted from a vibroacoustical coupled system are significant for predicting and improving the noise performance in fields such as automobiles [1], aeronautics and astronautics [2]. This specific vibroacoustical application pushes model identification into a more challenging battle, where not only the overall coupled system but also the decoupled structural/acoustic sub-model should be identified [3]. In practical application, the combined vibrational/acoustic frequency response function (FRF) is measured from the coupled system, and the modes extracted from the FRF are coupled by nature. However, it is significant for the engineer to decide each of the modes is governed by the structural part or acoustic part, such that each mode can be specifically managed to control the structural vibration or acoustical noise. The decoupling of vibroacoustics is critical as it provides not only a comprehensive understanding of the coupling mechanism but also a precise prediction on the specific structural/acoustic behavior. On the other hand, decoupling of vibroacoustics is important in terms of model validation. Decoupled models are often used in the design stage, either in vacuum structural analysis or rigid walled acoustic analysis. However, the practical measurement is always performed on the coupled system.

\footnotetext{
*Corresponding author.

Email addresses: sifeng.bi@femto-st.fr (S. Bi); morvan.ouisse@femto-st.fr (M. Ouisse);

emmanuel.foltete@femto-st.fr (E. Foltete); arnaud.jund@mpsa.com (A. Jund)
} 
Hence being able to represent the decoupled behavior from the coupled measurement is the key preparation for validation of the numerical models used for the design.

As a promising identification technique, the properness enforcement method has been developed in the literature for both symmetric and non-symmetric systems. The concept of properness condition is described with various names in different literatures [4-6]. Ideally, this condition is automatically fulfilled by the exact complex modes of the system without noise. In real applications, however, the noised modes must be corrected to make sure the properness condition is enforced, so that the modes can be safely used to reconstruct the system matrices. Balmès [7] developed a method to enforce this properness condition in structural dynamics, which has been recently extended to non-symmetrical problems [8] and the specific vibroacoustical applications [9]. However, there is still a remarkable obstacle when the engineer attempts to apply this method on real vibroacoustical problems. This obstacle arises from the following four aspects.

First, the vibroacoustical coupling implies the acoustic and structural responses are dependent with each other, hence the coupled behavior must be analyzed as one unity $[10,11]$. This consideration leads to a problem which exists only in vibroacoustics: the correlation between the coupled and decoupled models. Assume that $n$ coupled modes are identified from the measurement. Each mode, exhibiting a coupled behavior, founds its origin either in the acoustic part or in the structural part of the system. In the current procedure, the engineer must assign the nature of each mode (structural/acoustic) from the coupled mode list. An incorrect judgment of the configuration obviously leads to unphysical degree of freedoms (dofs) of the structural/acoustic sub-model and, furthermore, leads to redundant or missed decoupled modes. In this context, the term correlation explicitly refers to the procedure to decide the physical number of the structural/acoustic modes. This problem should be the primary consideration in vibroacoustical identification, but the published research on this issue is unfortunately limited. Note that the correlation in this context is different with the term described in Ref [9] whose purpose is to find the best sensors and actuators placement for modal analysis. Other literatures in model updating $[12,13]$ and validation $[14,15]$ refer to correlation as the process of checking the spatial coincidence between two vectors, which is also different with the correlation considered herein.

Second, compared with the structural dynamics, the vibroacoustical system behavior has more challenging characteristics: 1) The value of acoustical response is several orders of magnitude larger than the structural response, implying that the system response is quite sensitive to the noise; 2) The coupling between the acoustic and structural parts as well as the damping terms $[16,17]$ of the system are more complex and more difficult to be correctly represented; 3) Hard-to-control factors (e.g. sealing effectiveness and microphone sensitivity) lead the vibroacoustical experiment operationally more complex than the traditional structural experiments, introducing more noise in the measurement [18]. These difficulties hinder the properness enforcement method from providing precise results, especially in complex cases such as strong coupling or closed modes in given frequency range. Appropriate techniques besides the properness enforcement method are desired to optimize the modes before they can be safely used to construct the system matrices.

Third, vibroacoustics leads to non-symmetrical formulations in modal analysis, which require specific extensions against the classical techniques. The corresponding non-symmetric extensions are described in Ref. [8], however, explicit equation development for the vibroacoustical eigenvectors is still absent in the literature. Consequently, this paper proposes an extended least-square complex frequency-domain (LSCF) method which provides the calculation equations for the right and left eigenvectors during vibroacoustical identification.

The last aspects, for cases with large number of sensors in the experiment, the dofs of the model is too large, leading to a huge calculation burden for its further application. Being able to identify the models with a smaller size, i.e. with reduced dofs, is another common and important aspect in model identification [19, 20]. 
The objective of this work is to build mass, stiffness and damping matrices of an equivalent system which is capable of representing the same behavior as the experimentally measured one, but also to have the ability to be decoupled, which means that structural and acoustics effects can be investigated independently Considering the above mentioned difficulties, an integrated approach containing three aspects, i.e. identification, optimization and correlation, is proposed to overcome the obstacle in vibroacoustical identification.

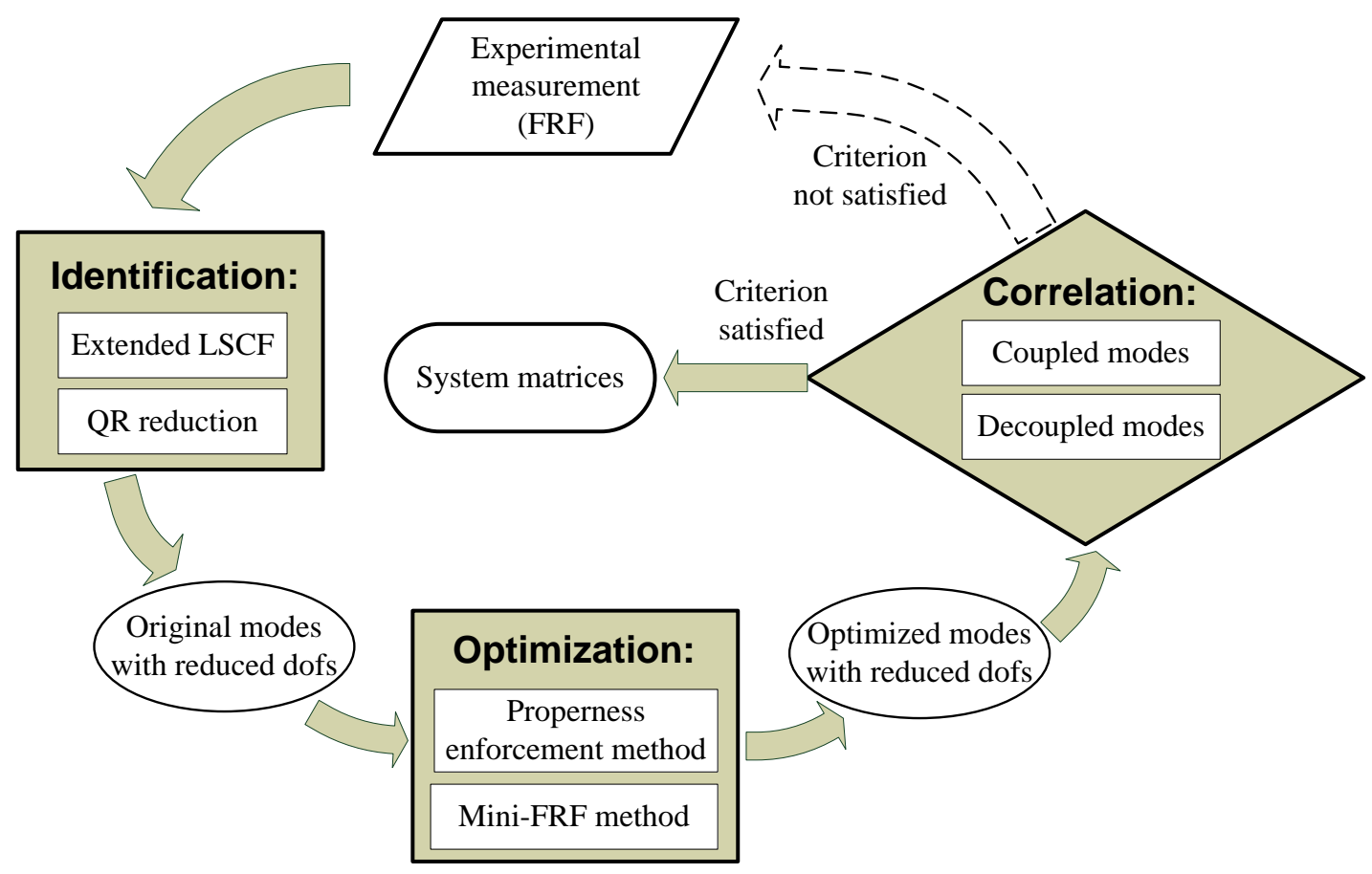

Fig. 1: The integrated framework for vibroacoustical identification

As shown in Fig. 1, this framework starts directly from the experimental measurements, from which the complex modes are identified by an extended LSCF method. A reduction technique based on the QR decomposition is subsequently executed on the complex modes. The reconstruction procedure from the complex modes to the system matrices, i.e. the inverse procedure, is quite sensitive to the noise, leading to large changes in the represented system behavior. Consequently, an optimization procedure is critical before the inverse procedure. Two optimal methods are employed, respectively the properness enforcement method and the "minimum FRF" method. The properness enforcement method has been described in Ref. [9], while the alternative minimum FRF method is proposed here as a necessary supplement especially in practical applications where more noise and strong coupling effects can occur. The last aspect of this framework is correlation, which is a specially required procedure for the vibroacoustical application. A discrimination technique based on the classical modal assurance criterion (MAC) [21] is proposed to filter the redundant modes, so that the physical configuration of the structural and acoustic dofs is obtained in the finally identified model. In case the criterion is not satisfied during correlation, a new judgement of the structural/acoustic dofs is proposed and a new round of identification is executed as shown in Fig. 1.

This paper is organized as follows. Section 2 is about the initial identification procedure where the specific formulation of vibroacoustics and the extended LSCF method are described. Section 3 recalls the properness enforcement method and proposes the minimum FRF method which is employed in the optimization procedure. Section 4 introduces two specially designed techniques, i.e. the QR decomposition reduction method and the MAC correlation procedure. Two case studies are given in Section 5. The simulated case study is presented to illustrate each aspect of the approach in detail so that the reader can reproduce the result. The 
following experimental case study is given to demonstrate the feasibility in practical application with emphases on noise and strong coupling effects. Finally, Section 6 presents the conclusions and perspectives of this work.

\section{Formulation of vibroacoustics and identification of complex modes}

\subsection{Vibroacoustical second-order problem}

The classical second-order formulation of the structural dynamics is

$$
\mathbf{M} \ddot{\mathbf{q}}(t)+\mathbf{C} \dot{\mathbf{q}}(t)+\mathbf{K q}(t)=\mathbf{f}(t)
$$

where $\mathbf{q}(t)$ is the system response; $\mathbf{f}(t)$ is the excitation vector; $\mathbf{M}, \mathbf{C}$, and $\mathbf{K}$ are respectively the mass, damping, and stiffness matrices. All the system matrices are assumed to be real, but as for the vibroacoustical problem considered herein, they are not necessarily symmetric.

A fluid domain (i.e. cavity) surrounded by an elastic domain is typically proposed to represent the vibroacoustical system. The response is described based on natural fields which are directly measured from different domains, i.e. displacement of the structure and acoustic pressure of the cavity. Then the system has a specific topology as

$$
\left[\begin{array}{cc}
\mathbf{M}_{s} & \mathbf{0} \\
\mathbf{L}^{T} & \mathbf{M}_{a}
\end{array}\right]\left\{\begin{array}{c}
\ddot{\mathbf{x}}(t) \\
\ddot{\mathbf{p}}(t)
\end{array}\right\}+\left[\begin{array}{cc}
\mathbf{C}_{s} & \mathbf{0} \\
\mathbf{0} & \mathbf{C}_{a}
\end{array}\right]\left\{\begin{array}{c}
\dot{\mathbf{x}}(t) \\
\dot{\mathbf{p}}(t)
\end{array}\right\}+\left[\begin{array}{cc}
\mathbf{K}_{s} & -\mathbf{L} \\
\mathbf{0} & \mathbf{K}_{a}
\end{array}\right]\left\{\begin{array}{c}
\mathbf{x}(t) \\
\mathbf{p}(t)
\end{array}\right\}=\left\{\begin{array}{c}
\mathbf{F}_{s}(t) \\
\dot{\mathbf{Q}}_{a}(t)
\end{array}\right\}
$$

where $\mathbf{x}(t)$ is the displacement of the structure, $\mathbf{p}(t)$ is the acoustic pressure of the cavity, $\mathbf{F}_{s}(t)$ is the excitation on the structure, $\dot{\mathbf{Q}}_{a}(t)$ is the acoustic excitation source (volume acceleration [10]) in the cavity. $\mathbf{M}_{s}$ and $\mathbf{K}_{s}$ are respectively the mass and stiffness matrices of the structure; $\mathbf{M}_{a}$ and $\mathbf{K}_{a}$ are respectively the mass and stiffness matrices of the cavity; $\mathbf{C}_{s}$ and $\mathbf{C}_{a}$ are respectively the structural and acoustic losses; $\mathbf{L}$ is the coupling matrix.

The right and left eigenvectors are required to describe the associated non-symmetrical quadratic eigenvalue problem:

$$
\begin{aligned}
& \left(\mathbf{M} \lambda_{i}^{2}+\mathbf{C} \lambda_{i}+\mathbf{K}\right) \boldsymbol{\varphi}_{R i}=0 \\
& \boldsymbol{\varphi}_{L i}^{T}\left(\mathbf{M} \lambda_{i}^{2}+\mathbf{C} \lambda_{i}+\mathbf{K}\right)=0
\end{aligned}
$$

where $\lambda_{j}, i=1,2, \cdots, n$, is the $i$-th eigenvalue associated to the $i$-th right and left eigenvector $\boldsymbol{\varphi}_{R i}$ and $\boldsymbol{\varphi}_{L i}$; and $n$ is the total number of dofs.

The specific topology of the system matrices in Eq. (2) involves the hypothesis that there is no loss at the coupling between the structural and acoustic parts, and the internal losses can be represented using equivalent viscous models. A detailed description of the vibroacoustical formulation and its damping conditions is given by Ref. [9].

\subsection{State-space representation}

The non-symmetrical nature of the vibroacoustics implies that the right and left modes must be described separately. The state-space representation of Eq. (1) is

$$
\left[\begin{array}{cc}
\mathbf{C} & \mathbf{M} \\
\mathbf{M} & \mathbf{0}
\end{array}\right]\left\{\begin{array}{c}
\ddot{\mathbf{q}}(t) \\
\dot{\mathbf{q}}(t)
\end{array}\right\}-\left[\begin{array}{cc}
-\mathbf{K} & \mathbf{0} \\
\mathbf{0} & \mathbf{M}
\end{array}\right]\left\{\begin{array}{c}
\dot{\mathbf{q}}(t) \\
\mathbf{q}(t)
\end{array}\right\}=\left\{\begin{array}{c}
\mathbf{f}(t) \\
0
\end{array}\right\} .
$$

The corresponding eigenvalue problem is then represented as 


$$
\begin{gathered}
\left(\left[\begin{array}{cc}
\mathbf{C} & \mathbf{M} \\
\mathbf{M} & \mathbf{0}
\end{array}\right] \lambda_{i}-\left[\begin{array}{cc}
-\mathbf{K} & \mathbf{0} \\
\mathbf{0} & \mathbf{M}
\end{array}\right]\right)\left\{\begin{array}{c}
\boldsymbol{\varphi}_{R i} \\
\boldsymbol{\varphi}_{R i} \lambda_{i}
\end{array}\right\}=\mathbf{0} \\
\left\{\begin{array}{c}
\boldsymbol{\varphi}_{L i} \\
\boldsymbol{\varphi}_{L i} \lambda_{i}
\end{array}\right\}^{T}\left(\left[\begin{array}{cc}
\mathbf{C} & \mathbf{M} \\
\mathbf{M} & \mathbf{0}
\end{array}\right] \lambda_{i}-\left[\begin{array}{cc}
-\mathbf{K} & \mathbf{0} \\
\mathbf{0} & \mathbf{M}
\end{array}\right]\right)=\mathbf{0} .
\end{gathered}
$$

The eigenvalues are stored in the spectral matrix $\Lambda=\left[\ddots^{\cdot} \lambda_{j} \cdot.\right]$, then the matrix form of the eigenvectors are expressed as

$$
\Theta_{R}=\left[\begin{array}{c}
\Phi_{R} \\
\Phi_{R} \boldsymbol{\Lambda}
\end{array}\right], \quad \Theta_{L}=\left[\begin{array}{c}
\Phi_{L} \\
\Phi_{L} \boldsymbol{\Lambda}
\end{array}\right] .
$$

Supposing $\mathbf{U}=\left[\begin{array}{cc}\mathbf{C} & \mathbf{M} \\ \mathbf{M} & \mathbf{0}\end{array}\right]$ and $\mathbf{A}=\left[\begin{array}{cc}-\mathbf{K} & \mathbf{0} \\ \mathbf{0} & \mathbf{M}\end{array}\right]$, then Eq. (5) is simply rewritten as

$$
\begin{aligned}
\mathbf{U} \Theta_{R} \boldsymbol{\Lambda} & =\mathbf{A} \Theta_{R} \\
\mathbf{U}^{T} \Theta_{L} \boldsymbol{\Lambda} & =\mathbf{A}^{T} \Theta_{L}
\end{aligned}
$$

Let $\xi=\left[\ddots \xi_{j} \cdot.\right]$ denote a diagonal matrix with $n$ arbitrary diagonal elements, the orthogonality between different modes is represented as

$$
\Theta_{L}^{T} \mathbf{U} \Theta_{R}=\xi \text { or } \Theta_{L}^{T} \mathbf{A} \Theta_{R}=\xi \Lambda .
$$

In the following context, it is important to note the arbitrary diagonal matrix $\xi$ is normalized as $\xi=\mathbf{E}_{n}$ where $\mathbf{E}_{n}$ is the $n$-by- $n$ identity matrix. This normalization condition is significant in the following inverse procedure to properly reconstruct the system matrices.

For a general non-symmetrical problem, the right and left eigenvectors are different and have no certain relationship. While in this vibroacoustical case, due to the specific topology in Eq. (2), there is a direct link between the right and left modes [10]:

$$
\text { If } \Phi_{R}=\left[\begin{array}{l}
\mathbf{X} \\
\mathbf{P}
\end{array}\right] \text {, then } \Phi_{L}=\left[\begin{array}{c}
\mathbf{X} \\
-\mathbf{P} \mathbf{\Lambda}^{-2}
\end{array}\right]
$$

where $\mathbf{X}$ is the sub-eigenvector corresponding to the structural dofs; $\mathbf{P}$ is the sub-eigenvector corresponding to the acoustic dofs. Note that this relationship is valid under the situation with only viscous damping and without structural-acoustic cross damping [9]. This hypothesis is fundamental for the vibroacoustical problem considered herein and it is also important for the extended LSCF method in the following section.

\subsection{Extension of the LSCF identification method}

The classical LSCF method has been demonstrated as a reliable technique to identify the complex modes from experimental measurements $[22,23]$. As this method is initially developed for symmetrical systems, an extension is required for this non-symmetrical case. Based on the complex curve-fitting of the FRF, the first step is to identify the complex poles (i.e. eigenvalues $\lambda_{i}$ ). This can be done in exactly the same way as if the system were symmetrical, since the eigenvalues associated with the right and left eigenvectors are equal. Once the poles have been identified, the right and left eigenvectors are calculated based on the so-called residue matrix. Construction of the residue matrix is relative to the position of the excitation. Assume the excitation is applied on the $k$-th dof, the residue matrix is

$$
\mathbf{R}_{k}=\Phi_{R} \mathbf{D}_{k}
$$

where $\Phi_{R}$ is the unknown right eigenvector matrix; $\mathbf{D}_{k}$ is the modal participation matrix, which is diagonal 
and whose terms are the $k$-th row of the unknown left eigenvector matrix. This indicates each column of $\mathbf{R}_{k}$ is proportional to a right eigenvector. Note that depending if the excitation is applied on a structural or acoustic dof, the proportionality coefficients in $\mathbf{R}_{k}$ are different, and consequently the calculation of eigenvectors belongs to two situations.

\section{- When the excitation is applied on a structural dof:}

Eq. (10) is rewritten as

$$
\begin{aligned}
\mathbf{R}_{k} & =\left[\begin{array}{lll}
\cdots & \boldsymbol{\varphi}_{R i} & \cdots
\end{array}\right]\left[\begin{array}{lll}
\ddots & \\
x_{k i} & \\
& \ddots
\end{array}\right] \\
& =\left[\begin{array}{llll}
\boldsymbol{\varphi}_{R 1} x_{k 1} & \boldsymbol{\varphi}_{R 2} x_{k 2} & \cdots & \boldsymbol{\varphi}_{R n} x_{k n}
\end{array}\right]
\end{aligned}
$$

where $\boldsymbol{\varphi}_{R i}$ is the $i$-th right eigenvector; $x_{k i}$ is the unknown coefficient. As $x_{k i}$ is simultaneously the element in the $k$-th row of the left eigenvector matrix which corresponds to the structural dof, it can be evaluated from the residue matrix by

$$
x_{k i}=\sqrt{\mathbf{R}_{k}(k, i)}, \forall i=1, \cdots, n
$$

where $\mathbf{R}_{k}(k, i)$ is the element positioned at the $k$-th row and $i$-th column in matrix $\mathbf{R}_{k}$. Then the right eigenvector is extracted as

$$
\boldsymbol{\varphi}_{R i}=\frac{\mathbf{R}_{k}(:, i)}{x_{k i}}, \forall i=1, \cdots, n
$$

where $\mathbf{R}_{k}(:, i)$ is the $i$-th column of $\mathbf{R}_{k}$, afterwards the left eigenvector is calculated following Eq. (9).

\section{- When the excitation is applied on an acoustic dof:}

In this case, Eq. (10) should be rewritten in a different way compared with Eq. (11):

$$
\begin{aligned}
\mathbf{R}_{k} & =\left[\begin{array}{lll}
\cdots & \boldsymbol{\varphi}_{R i} & \cdots
\end{array}\right]\left[\begin{array}{lll}
\ddots & & \\
& -p_{k i} \lambda_{i}^{-2} & \ddots
\end{array}\right] \\
& =\left[\begin{array}{lllll}
-\boldsymbol{\varphi}_{R 1} p_{k 1} \lambda_{1}^{-2} & -\boldsymbol{\varphi}_{R 2} p_{k 2} \lambda_{2}^{-2} & \cdots & -\boldsymbol{\varphi}_{R n} p_{k n} \lambda_{n}^{-2}
\end{array}\right]
\end{aligned}
$$

where $p_{k i}$ is the unknown coefficient with the same sense as $x_{k i}$ in Eq. (11). $p_{k i}$ is simultaneously the element in the $k$-th row of the left eigenvector, but herein it corresponds to the acoustic dof. Consequently, $p_{k i}$ is calculated as

$$
p_{k i}=\sqrt{-\mathbf{R}_{k}(k, i) \lambda_{i}^{2}}, \forall i=1, \cdots, n .
$$

Then the right eigenvector is extracted as

$$
\boldsymbol{\varphi}_{R i}=-\frac{\mathbf{R}_{k}(:, i)}{p_{k i} \lambda_{i}^{2}}, \forall i=1, \cdots, n .
$$

Similarly, the left eigenvector can be obtained following Eq. (9).

\section{Optimization of the identified complex modes}

Identified from the measurements, the complex modes are unavoidably polluted by noise, which comes from both the measurements and the identification process. The optimization procedure is required before the directly identified modes can be safely utilized to reconstruct the system matrices. The reconstruction procedure is derived from the orthogonality relationship in Eq. (8). When $\xi$ is normalized as $\mathbf{E}_{n}$ (the normalization condition), the inverse of $\mathbf{U}$ and $\mathbf{A}$ are obtained as 


$$
\begin{aligned}
& \mathbf{U}^{-1}=\boldsymbol{\Theta}_{R} \boldsymbol{\Theta}_{L}^{T} \\
& \mathbf{A}^{-1}=\boldsymbol{\Theta}_{R} \boldsymbol{\Lambda} \boldsymbol{\Theta}_{L}^{T}
\end{aligned}
$$

Recalling Eq. (6), the above equations is rewritten as

$$
\begin{aligned}
& {\left[\begin{array}{cc}
\mathbf{C} & \mathbf{M} \\
\mathbf{M} & 0
\end{array}\right]^{-1}=\left[\begin{array}{cc}
0 & \mathbf{M}^{-1} \\
\mathbf{M}^{-1} & -\mathbf{M}^{-1} \mathbf{C M}^{-1}
\end{array}\right]=\left[\begin{array}{cc}
\Phi_{R} \Phi_{L}^{T} & \Phi_{R} \boldsymbol{\Lambda} \Phi_{L}^{T} \\
\Phi_{R} \boldsymbol{\Lambda} \Phi_{L}^{T} & \Phi_{R} \boldsymbol{\Lambda}^{2} \Phi_{L}^{T}
\end{array}\right]} \\
& {\left[\begin{array}{cc}
-\mathbf{K} & 0 \\
0 & \mathbf{M}
\end{array}\right]^{-1}=\left[\begin{array}{cc}
-\mathbf{K}^{-1} & 0 \\
0 & \mathbf{M}^{-1}
\end{array}\right]=\left[\begin{array}{cc}
\Phi_{R} \boldsymbol{\Lambda}^{-1} \Phi_{L}^{T} & \Phi_{R} \Phi_{L}^{T} \\
\Phi_{R} \boldsymbol{\Lambda} \Phi_{L}^{T} & \Phi_{R} \boldsymbol{\Lambda} \Phi_{L}^{T}
\end{array}\right] .}
\end{aligned}
$$

Then the system matrices are extracted as

$$
\begin{aligned}
& \mathbf{M}=\left[\Phi_{R} \boldsymbol{\Lambda} \Phi_{L}^{T}\right]^{-1} \\
& \mathbf{K}=-\left[\Phi_{R} \boldsymbol{\Lambda}^{-1} \Phi_{L}^{T}\right]^{-1} \\
& \mathbf{C}=-\left[\mathbf{M} \Phi_{R} \boldsymbol{\Lambda}^{2} \Phi_{L}^{T} \mathbf{M}\right] .
\end{aligned}
$$

The above inverse procedure is valid only if the so-called properness condition is satisfied, which can be easily yielded from Eq. (18) as

$$
\Phi_{R} \Phi_{L}^{T}=0
$$

Note that the above properness condition is universal for all non-symmetrical problems. For the particular vibroacoustical case, the properness condition can be rewritten only using the right eigenvector as

$$
\left[\begin{array}{ll}
\mathbf{X} \mathbf{X}^{T} & -\mathbf{X} \boldsymbol{\Lambda}^{-2} \mathbf{P}^{T} \\
\mathbf{P X} & -\mathbf{P} \Lambda^{-2} \mathbf{P}^{T}
\end{array}\right]=0
$$

where $\mathbf{X}$ and $\mathbf{P}$ have the same sense as in Eq. (9).

\subsection{Properness enforcement method}

Due to the noise, the identified complex modes generally do not verify the properness condition. Hence the eigenvectors should be corrected so that the condition is reinforced. The properness enforcement method has already been described in Ref. [9]. Considering theoretical integrity, this method is briefly recalled in this subsection. Note that this method is found to be unsteady in some complex applications (see Section 5). Consequently, the minimum FRF method is proposed as a necessary supplement in the following subsection.

The enforcement procedure is essentially an optimization problem: Find the approximate $\tilde{\mathbf{X}}$ and $\tilde{\mathbf{P}}$, minimizing $f_{1}=\|\tilde{\mathbf{X}}-\mathbf{X}\|$ and $f_{2}=\|\tilde{\mathbf{P}}-\mathbf{P}\|$, with the constraints as

$$
\left\{\begin{array}{l}
g_{1}=\tilde{\mathbf{X}} \tilde{\mathbf{X}}^{T}=0 \\
g_{2}=\tilde{\mathbf{X}} \tilde{\mathbf{P}}^{T}=0 \\
g_{3}=\tilde{\mathbf{X}} \Lambda^{-2} \tilde{\mathbf{P}}^{T}=0 \\
g_{4}=\tilde{\mathbf{P}} \Lambda^{-2} \tilde{\mathbf{P}}^{T}=0
\end{array}\right.
$$

To solve this problem, a hybrid matrix combining both the right and left eigenvectors is constructed as 


$$
\boldsymbol{\Psi}=\left[\begin{array}{c}
\mathbf{X} \\
\mathbf{P} \\
-\mathbf{P} \Lambda^{-2}
\end{array}\right]
$$

Considering the properness condition $\boldsymbol{\Psi} \boldsymbol{\Psi}^{T}=0$, one can get the constraints as

$$
\boldsymbol{\Psi} \boldsymbol{\Psi}^{T}=\left[\begin{array}{ccc}
\mathbf{X} \mathbf{X}^{T} & \mathbf{X} \mathbf{P}^{T} & -\mathbf{X} \boldsymbol{\Lambda}^{-2} \mathbf{P}^{T} \\
\mathbf{P} \mathbf{X}^{T} & \mathbf{P P}^{T} & -\mathbf{P} \Lambda^{-2} \mathbf{P}^{T} \\
-\mathbf{P} \boldsymbol{\Lambda}^{-2} \mathbf{X}^{T} & -\mathbf{P} \boldsymbol{\Lambda}^{-2} \mathbf{P}^{T} & \mathbf{P} \Lambda^{-4} \mathbf{P}^{T}
\end{array}\right]=0 .
$$

The usage of $\Psi$ simplifies the problem from a vibroacoustical case to the typically symmetrical case. The methodology proposed in Ref. [7] can be easily employed here by solving a Riccati equation. This equation is derived from the simplified optimization problem:

$$
\text { Find } \tilde{\boldsymbol{\Psi}} \text {, minimizing }\|\tilde{\boldsymbol{\Psi}}-\boldsymbol{\Psi}\| \text {, while } \tilde{\mathbf{\Psi}} \tilde{\mathbf{\Psi}}^{T}=0 \text {. }
$$

Solution of this problem is achieved by a Lagrange multiplier matrix $\boldsymbol{\delta}$ :

$$
\tilde{\mathbf{\Psi}}=\left[\mathbf{E}_{m}-\boldsymbol{\delta} \overline{\boldsymbol{\delta}}\right]^{-1}[\boldsymbol{\Psi}-\boldsymbol{\delta} \overline{\mathbf{\Psi}}]
$$

where $\mathbf{E}_{m}$ is the identity matrix; $m$ is the dofs in $\Psi$; the overbars denotes the complex conjugate. $\boldsymbol{\delta}$ is obtained by solving the Riccati equation:

$$
\boldsymbol{\Psi} \boldsymbol{\Psi}^{T}-\boldsymbol{\delta} \overline{\boldsymbol{\Psi}} \boldsymbol{\Psi}^{T}-\boldsymbol{\Psi} \overline{\boldsymbol{\Psi}}^{\mathrm{T}} \boldsymbol{\delta}+\boldsymbol{\delta} \overline{\boldsymbol{\Psi}} \overline{\boldsymbol{\Psi}}^{T} \boldsymbol{\delta}=0
$$

\subsection{Minimum FRF method}

An alternative method is to directly consider the relationship between the measured frequency response and the system matrices. In case the harmonic excitation is added on the system, Eq. (1) can be rewritten under the frequency domain:

$$
\left(-\omega^{2} \mathbf{M}+\mathrm{i} \omega \mathbf{C}+\mathbf{K}\right) \mathbf{G}=\mathbf{F}
$$

where $\mathbf{G}$ and $\mathbf{F}$ are respectively the complex amplitude of $\mathbf{q}(t)$ and $\mathbf{f}(t)$ in Eq. (1). Given a measured $\mathbf{G}$ associated with the excitation $\mathbf{F}$, the system matrices can be directly found by solving the minimization problem:

$$
\text { Find }(\tilde{\mathbf{M}}, \tilde{\mathbf{C}}, \tilde{\mathbf{K}}) \text {, minimizing }\left\|\left(-\omega^{2} \tilde{\mathbf{M}}+\mathrm{i} \omega \tilde{\mathbf{C}}+\tilde{\mathbf{K}}\right)^{-1} \mathbf{F}-\mathbf{G}\right\| \text {, while }(\tilde{\mathbf{M}}, \tilde{\mathbf{C}}, \tilde{\mathbf{K}}) \in \mathfrak{R}
$$

where $\mathfrak{R}$ is the space of admissible matrices fulfilling the specific vibroacoustical topology in Eq. (2). The objective function can be reorganized as a set of linear equations

$$
\hat{\mathbf{G}} \boldsymbol{\alpha}=\hat{\mathbf{F}}
$$

where $\hat{\mathbf{G}}$ is a reorganized matrix containing elements of $\boldsymbol{\omega}$ and $\mathbf{G} ; \boldsymbol{\alpha}$ is the unknown vector containing the elements extracted from the system matrices; $\hat{\mathbf{F}}$ is the reorganized vector containing the elements of $\mathbf{F}$. $\boldsymbol{\alpha}$ is determined by the pseudo-inverse of $\hat{\mathbf{G}}$ to achieve the least-square error,

$$
\boldsymbol{\alpha}=\left(\hat{\mathbf{G}}^{T} \hat{\mathbf{G}}\right)^{-1} \hat{\mathbf{G}} \hat{\mathbf{F}} .
$$

This method directly finds the matrices with the consideration to minimize the error between the simulated 
frequency response and measured frequency response, without calculating the complex modes. Obviously, it involves high calculation cost, especially when the system has a large number of dofs. In case of low order reduced models, this method can serve as an efficient supplement of the properness enforcement method.

\section{Correlation of the identified modes}

\subsection{QR decomposition for modal reduction}

The objective of model reduction is to find an equivalent model with reduced dofs, which continues to exhibit the same system behavior as the full size one. Ref. [24] describes a methodology based on QR decomposition to optimize the placement of sensors in experiments. Though with different motivations, the activity in Ref. [24] obeys the same logic as which proposed in this work, which is screening the "key" dofs to represent the system behavior. Under this sense, the QR decomposition is a suitable technique to achieve this objective.

In order to discriminate the key dofs, the identified full size eigenvector matrix is investigated. The idea is that the most linear independent rows in this matrix construct a reduced matrix which provides a MAC with minimized off-diagonal terms. In other words, these dofs are capable of representing the maximum number of modes. QR decomposition is proposed here to extract these rows from the original eigenvector matrix $\Phi$ :

$$
\Phi^{T} \mathbf{E}=\mathbf{Q R}
$$

where $\mathbf{Q}$ is an orthogonal matrix; $\mathbf{R}$ is an upper triangular matrix; $\mathbf{E}$ is a column permutation matrix with the purpose of making the diagonal terms of $\mathbf{R}$ rank in descending order. The row vectors with the most significant independence are then discriminated according to the rearranged column number in matrix $\mathbf{E}$.

Two principles are fulfilled by this technique when reducing the dofs: i) The selected dofs are sensitive to the maximum number of modes in the frequency range of interest; ii) The system response on each selected dof is different (i.e. independent) enough compared with other dofs, allowing the maximum number of modes to be distinguishable. This is the reason why the technique is not only feasible for optimizing the placement of sensors before the experiment, but also for reducing a large size original model after the experiment. The MAC matrix of the reduced model can be employed to evaluate the effect of this technique by checking if the off-diagonal terms are minimized.

\subsection{Correlation between the coupled and decoupled model}

A special problem of vibroacoustical identification is to decide the right number of the structural/acoustic modes from the coupled mode list. The number of the coupled modes is $n$ :

$$
n=n_{s}+n_{a}
$$

where $n_{\mathrm{s}}$ and $n_{\mathrm{a}}$ are respectively the number of the structural and acoustic modes. For a fixed $n$, this paper proposes configuration to denote different choices of $n_{s}+n_{a}$. An incorrect configuration will lead to redundant or missed modes of the decoupled model. In this context, the term correlation is explicitly referring to the procedure to decide the physical configuration.

Before correlation, it is necessary to make clear the concepts between the coupled mode and the decoupled mode. The coupled mode associates with the coupled system matrices with the size as $n$-by- $n$. Because the coupled system matrices are reconstructed following the hypothetical topology, the decoupled matrices ( $\mathbf{M}_{s}$, $\mathrm{C}_{s}, \mathbf{K}_{s}$ and $\mathbf{M}_{a}, \mathbf{C}_{a}, \mathbf{K}_{a}$ ) are easily extracted following Eq. (2). The decoupled natural frequencies and eigenvectors are then calculated based on these decoupled matrices with the size as $n_{s}$-by- $n_{s} / n_{a}$-by- $n_{a}$. 
The proposed correlation procedure contains two aspects. The first aspect is based on the natural frequency, and secondly the eigenvector. The general idea is that a physical decoupled mode should be steady in different configurations. In other words, if there is a similar frequency value continually appears in different configurations, this frequency is assumed to reveal a physical mode.

Correlation of the eigenvector is performed by the MAC matrix between the decoupled and coupled eigenvectors. The crucial point for this comparison is to choose the suitable data to calculate the MAC. As the correlation procedure should be separately executed on the structural and acoustic parts, acoustic correlation is taken as an example in the following description.

The decoupled acoustic eigenvector matrix $\Phi_{a}$ is calculated from the acoustic matrices, with the size as $n_{a}$-by- $n_{a}$ :

$$
\Phi_{a}=\left[u_{1}, u_{2}, \ldots, u_{n_{a}}\right]
$$

where $u_{i}, \forall i=1, \ldots, n_{a}$, is the $i$-th acoustic eigenvector. Recalling Eq. (9), $\mathbf{P}$ is the coupled eigenvector matrix but only corresponding to the acoustic dofs. Clearly, $\mathbf{P}$ has the size as $n_{a}$-by- $n$, and it is constructed as

$$
\mathbf{P}=\left[v_{1}, v_{2}, \ldots, v_{n}\right] .
$$

where $v_{i}, \forall i=1, \ldots, n$, is the $i$-th coupled eigenvector but only corresponding to the acoustic dofs. Then the correlation MAC is calculated as

$$
\operatorname{MAC}_{i j}\left(\Phi_{a}, \mathbf{P}\right)=\frac{\left(u_{i}^{T} v_{j}\right)^{2}}{\left(u_{i}^{T} u_{i}\right)\left(v_{j}^{T} v_{j}\right)} ; \forall i=1, \ldots, n_{a} ; j=1, \ldots, n .
$$

Each configuration has its unique MAC matrix with the size as $n_{a}$-by- $n$. The MACs are evaluated focusing on each row which corresponds to each decoupled acoustic mode. The principles are: 1) The physical decoupled eigenvector should steady for different configurations (i.e. in different MACs). The term "steady rows" denotes rows with exactly the same elements, or at least, the rows are proportional with each other. 2) The physical acoustic eigenvector should only depend on its corresponding coupled eigenvector and be independent to all the other coupled eigenvectors. Consequently, a physical decoupled mode behaves as a unique row where only one element is equal to unity while others are close to zero.

Correlation based on natural frequency and eigenvector should be performed together so that a robust conclusion is obtained. Usage and feasibility of this correlation procedure is demonstrated in the following case studies.

\section{Case studies}

\subsection{The simulated case with large size matrices}

The overall approach is firstly illustrated by a simulated case study. The FRFs are not measured from an experiment, but calculated from per-defined system matrices. The matrices are extracted from a virtual hexahedral cavity $\left(780 \times 850 \times 600 \mathrm{~mm}^{3}\right)$ whose top surface is covered by a $4 \mathrm{~mm}$ thickness aluminum plate $\left(780 \times 850 \mathrm{~mm}^{2}\right)$ and other five surfaces are surrounded by rigid walls. Bulk modulus and density of the air inside the cavity are respectively $1.42 \times 10^{5} \mathrm{~Pa}$ and $1.225 \mathrm{~kg} / \mathrm{m}^{3}$; elastic modulus and density of aluminum are respectively $7.2 \times 10^{10} \mathrm{~Pa}$ and $2.7 \times 10^{3} \mathrm{~kg} / \mathrm{m}^{3}$. The illustration follows the key aspects: 
1. Identification of the original data: the starting point is the system matrices following the specific vibroacoustical topology. The theoretical FRF and natural frequencies are calculated based on these matrices. Identification is performed on the theoretical FRF, and the identified data is termed as "original" data which is served as reference in the following procedure.

2. Reduction: The complex eigenvectors are reduced from the original size to a much smaller size using the QR decomposition technique introduced in Section 4.1.

3. The direct data: the reduced modes are then utilized in the inverse procedure to reconstruct the system matrices, based on Eq. (19). These matrices are termed as "direct" because they are obtained from the modes without optimization. The direct FRF and frequencies are calculated based on the direct matrices.

4. The optimized data: the properness enforcement method and the minimum FRF method are respectively employed to obtain the optimized data, which are respectively termed as "proper" and "mini-frf" data.

5. Correlation: the correlation is performed on different configurations of the structural and acoustic dofs. The decoupled and reduced model with the physical configuration is finally obtained.

\subsubsection{Identification and reduction of the original data}

The proposed vibroacoustical system contains 130 structural dofs and 123 acoustic dofs, therefore the per-defined system matrices have the size as 253-by-253. The theoretical FRFs are calculated by adding a unit "acoustic force", which is expressed in term of acoustic volume acceleration with the unit as $\mathrm{m}^{3} / \mathrm{s}^{2}[10]$. An identification procedure based on the extended LSCF method is performed, as shown in Fig. 2. This identification procedure is performed in MODAN, which is an integrated structural dynamic identification software developed by Femto-ST Institute.

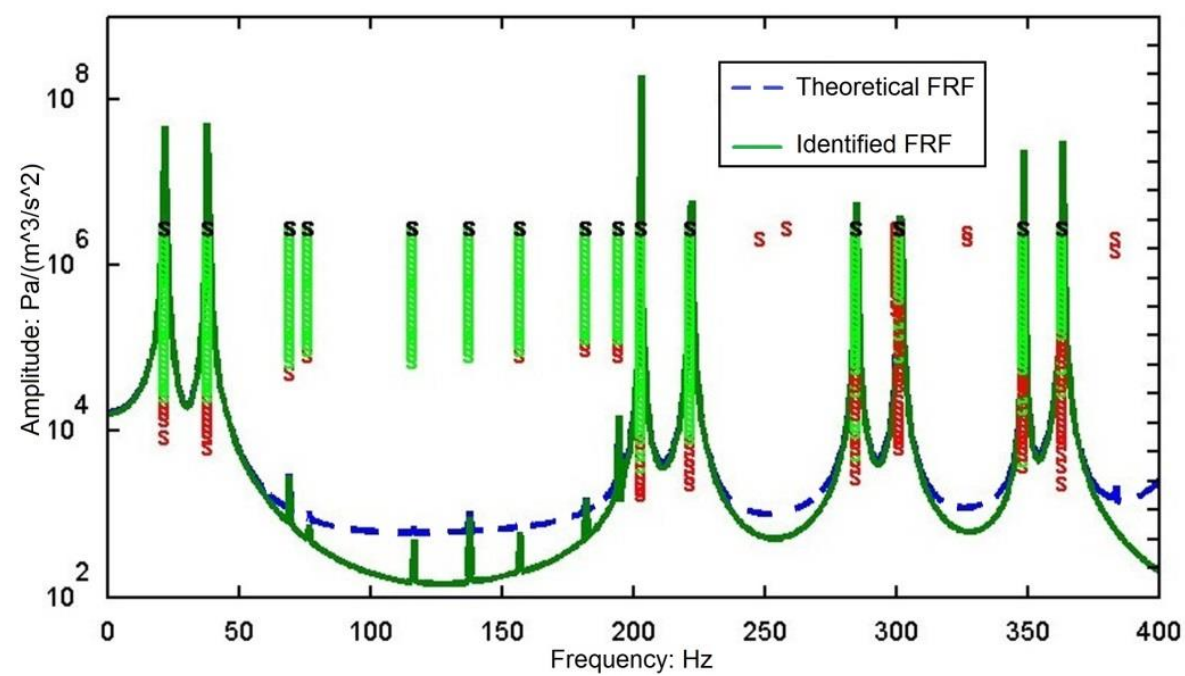

Fig. 2: The identification procedure and selection of the poles in MODAN

Note that there are differences between the theoretical FRF and the original FRF. The theoretical FRF is calculated from the per-defined system matrices, while the original FRF is identified from MODAN based on the theoretical FRF. As shown in Fig. 3, the original (i.e. identified) FRF has lost some modes nearby $250 \mathrm{~Hz}$ and $400 \mathrm{~Hz}$ compared with the theoretical FRF. Explanation for this discrepancy can be checked in Fig. 2 where some discrete poles nearby $250 \mathrm{~Hz}$ and $400 \mathrm{~Hz}$ are not selected. As these modes have been lost in the first step of identification, it is natural that the identified FRF cannot exactly represent the theoretical FRF. This phenomenon of mode losing is more common in real experimental case when there is more uncontrollable noise in the measurements. A more obvious influence of this phenomenon is revealed in the next case study. 


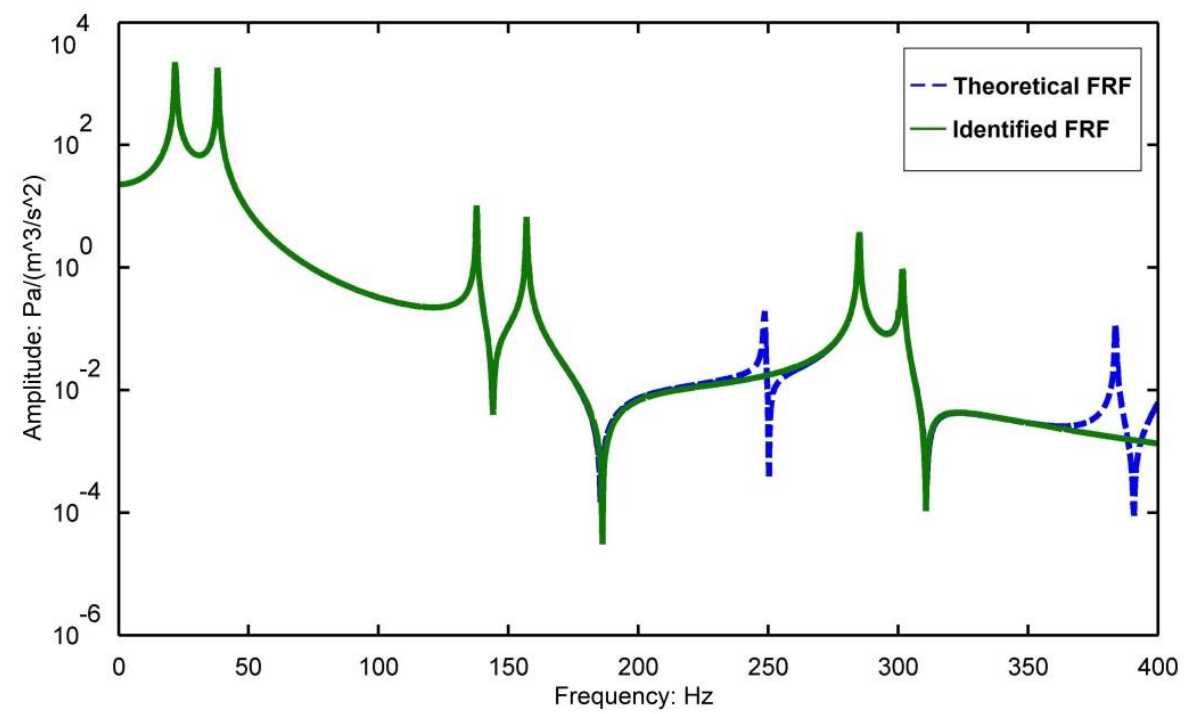

Fig. 3: The theoretical and identified (original) FRFs in MODAN

The frequency range considered herein is $0-400 \mathrm{~Hz}$ where 15 modes are identified. The QR decomposition is utilized to reduce the size of the eigenvectors from 253 to 15 . Before performing the reduction, it is important to make clear that each coupled mode corresponds to the structural part or acoustic part. The exact number of structural/acoustic modes among these 15 coupled modes is important for determining how many structural/acoustic dofs should be reserved in the reduced model. Since the original system matrices are pre-defined in this simulated example, the theoretical coupled and decoupled frequencies can be exactly calculated, as detailed in Table 1. For clarity, the decoupled frequencies are placed in the same row as their corresponding coupled frequencies, and the decoupled acoustic modes and their corresponding coupled modes are marked as bold and italic.

Table 1: The theoretical natural frequencies calculated from the pre-defined matrices

\begin{tabular}{cccc}
\hline \multirow{2}{*}{ Mode No. } & \multicolumn{3}{c}{ Theoretical natural frequencies (Hz) } \\
\cline { 2 - 4 } & Coupled & Decoupled structural & Decoupled acoustic \\
\hline $\mathbf{1}$ & $\mathbf{2 1 . 7 9}$ & & $\mathbf{2 8 . 3 8}$ \\
$\mathbf{3}$ & 38.16 & 29.54 & \\
$\mathbf{4}$ & 69.35 & 69.79 & \\
$\mathbf{5}$ & 76.64 & 77.10 & \\
$\mathbf{6}$ & 116.42 & 116.76 & \\
$\mathbf{7}$ & 137.65 & 137.90 & \\
$\mathbf{8}$ & 157.07 & 157.35 & \\
$\mathbf{9}$ & 182.20 & 182.42 & $\mathbf{2 0 2 . 4 7}$ \\
$\mathbf{1 0}$ & 194.65 & 194.96 & $\mathbf{2 2 1 . 6 6}$ \\
$\mathbf{1 1}$ & $\mathbf{2 0 2 . 9 8}$ & & $\mathbf{2 8 3 . 9 4}$ \\
$\mathbf{1 2}$ & $\mathbf{2 2 2 . 0 3}$ & & $\mathbf{3 0 1 . 4 9}$ \\
$\mathbf{1 3}$ & $\mathbf{2 8 4 . 9 3}$ & & $\mathbf{3 4 8 . 4 9}$ \\
$\mathbf{1 4}$ & $\mathbf{3 0 1 . 7 0}$ & & $\mathbf{3 6 3 . 1 6}$ \\
$\mathbf{1 5}$ & $\mathbf{3 4 8 . 7 7}$ & & \\
\hline
\end{tabular}

As shown in Table 1, among these 15 modes there are actually 8 structural modes and 7 acoustic modes, indicating 8 structural dofs and 7 acoustic dofs should be reserved in the reduced model. As mentioned in 
Section 4.1, the MAC matrix can be utilized to evaluate the effect of reduction. Fig. 4 shows the MAC matrices of the reduced and original models, showing the degree of independence among the eigenvectors. Both MAC matrices in Fig. 4 correspond to the acoustic part of the system. The MAC in Fig. 4(a) results from the eigenvector matrix reduced by QR decomposition (termed as MAC_QR); the MAC in Fig. 4(b) is obtained from an eigenvector matrix whose 7 rows are randomly selected from the original 130 rows of the acoustic eigenvector matrix (termed as MAC_Random).

The diagonal terms of these two MAC matrices are naturally equal to one, while the off-diagonal terms of them are obviously different. The off-diagonal terms of MAC_QR are basically minimized to zero indicating a high degree of independence among the rows. Contrary, the off-diagonal terms of MAC_Random have different values from zero to one, which means some rows in the randomly reduced matrix are dependent with others, and consequently, they are not the dofs suitable enough to be reserved during model reduction.

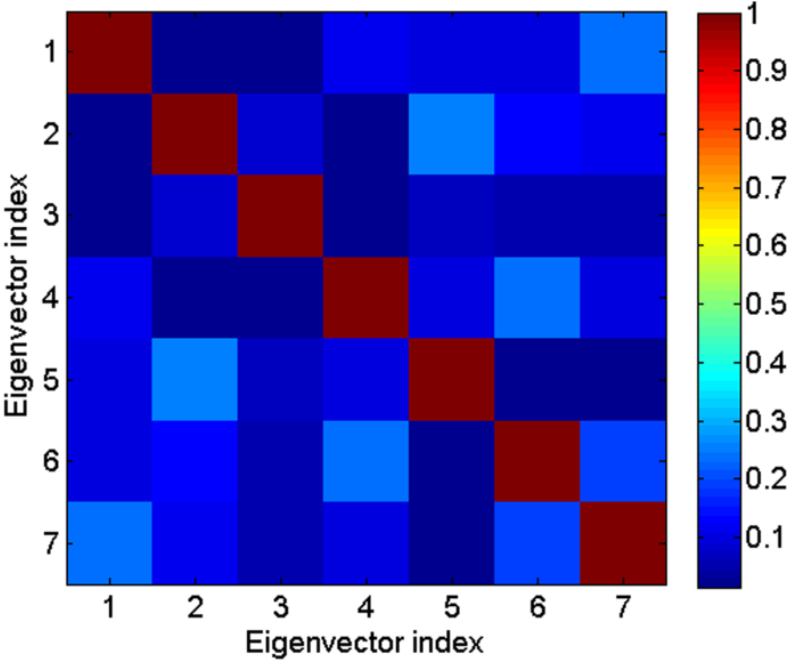

(a) MAC_QR

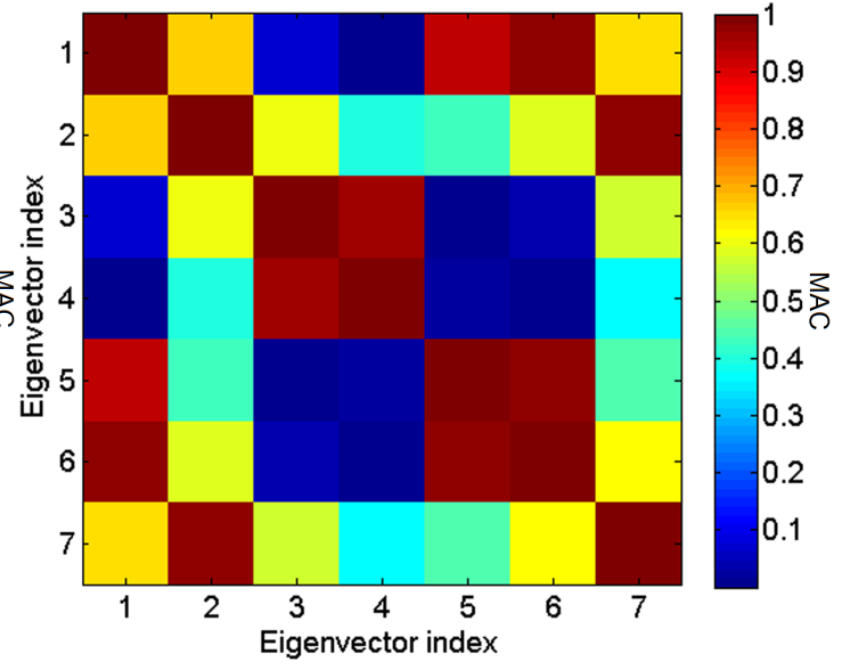

(b) MAC_Random

Fig. 4: MAC matrices of the QR reduced model (MAC_QR) and the randomly reduced model (MAC_Random)

\subsubsection{Comparison of the coupled data}

In practical applications where the exact number of structural and acoustic modes (i.e. $n_{s}$ and $n_{a}$ ) are unknown, all the possible configurations of " $n_{\mathrm{s}}+n_{\mathrm{a}}$ " should be evaluated. The configurations considered herein (respectively termed as Configs 1-4) are listed in Table 2. Undoubtedly different configurations bring different identification outcomes. For clarity, only the results of Config- 1 and Config- 2 are presented. The properness enforcement method is first executed, and the minimum FRF method is employed as a supplement on the specific part where the proper data is not perfect enough.

Table 2: Configurations of structural/acoustic dofs for the 15 coupled modes

\begin{tabular}{ccc}
\hline Configuration No. & $\boldsymbol{n}_{\boldsymbol{s}}$ & $\boldsymbol{n}_{\boldsymbol{a}}$ \\
\hline $\mathbf{1}$ & 6 & 9 \\
$\mathbf{2}$ & 7 & 8 \\
$\mathbf{3}$ & 8 & 7 \\
$\mathbf{4}$ & 9 & 6 \\
\hline
\end{tabular}

\section{- comparison of the proper FRFs}

The original, direct, and proper FRFs for Config-1 and Config-2 are respectively compared in Figs. 5 and 6. The proper FRFs for Config-1 fit with the original ones quite well on both structural and acoustic dofs. As the 
properness condition is not fulfilled by the direct data, the direct FRFs naturally fail to represent the original ones.

For Config-2, Fig. 6 shows the proper FRFs fail to represent most of the modes in range of 0-200 Hz, although they can fit with the original FRFs quite well in range of 200-400 Hz. Possible explanations for this discrepancy are:

1) The fact is most of the modes in the $0-200 \mathrm{~Hz}$ range are structural modes (recalling Table 1). The reserved structural dofs may be unphysical in Config-2, leading to a false representation of the structural modes. This implies a robust methodology for estimating the correct number of structural and acoustic dofs is necessary.

2) System responses of vibroacoustics have different units for different parts, i.e. $m$ for structural displacement and $\mathrm{Pa}$ for acoustic pressure. The absolute value of displacement is much smaller than acoustic pressure, implying the structural modes are more difficult to be handled especially when the structural dofs are unsuitably reduced. A "pre-adjustment" procedure is suggested to apply an amplification factor on the structural FRFs so that the FRFs of different dofs are adjusted to a similar scale. Even so, the difficulty remains in a certain dof (e.g. the $15^{\text {th }}$ dof in Figs. 5 and 6) where the structural resonances are much weaker than the acoustic ones.

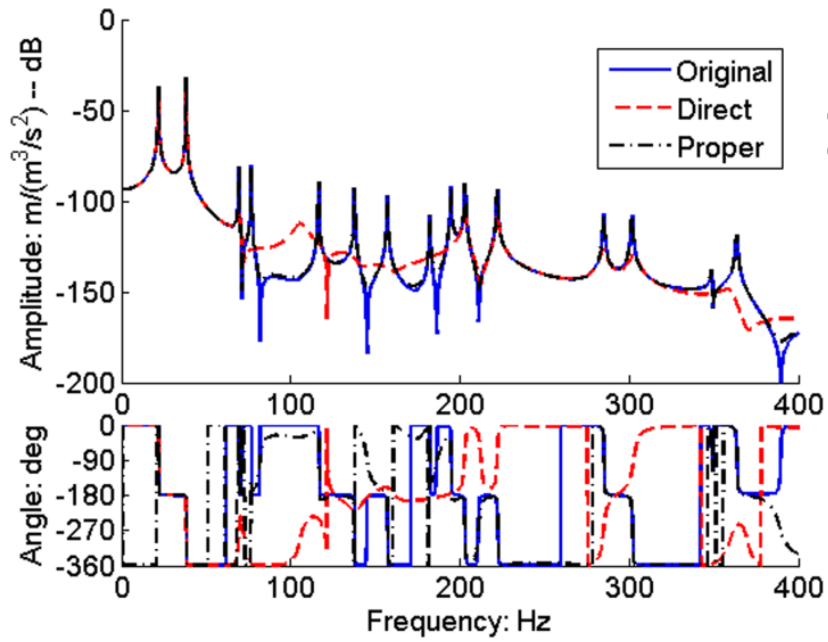

(a) the $5^{\text {th }}$ dof -- structural part

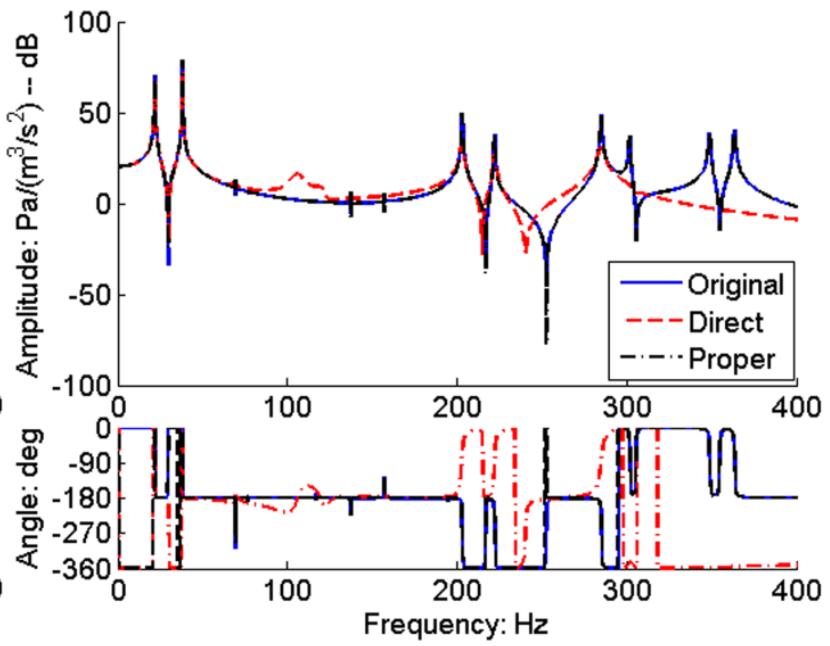

(b) the $15^{\text {th }}$ dof -- acoustic part

Fig. 5: The original, direct, and proper FRFs of the $5^{\text {th }}$ and $15^{\text {th }}$ dofs for Config-1

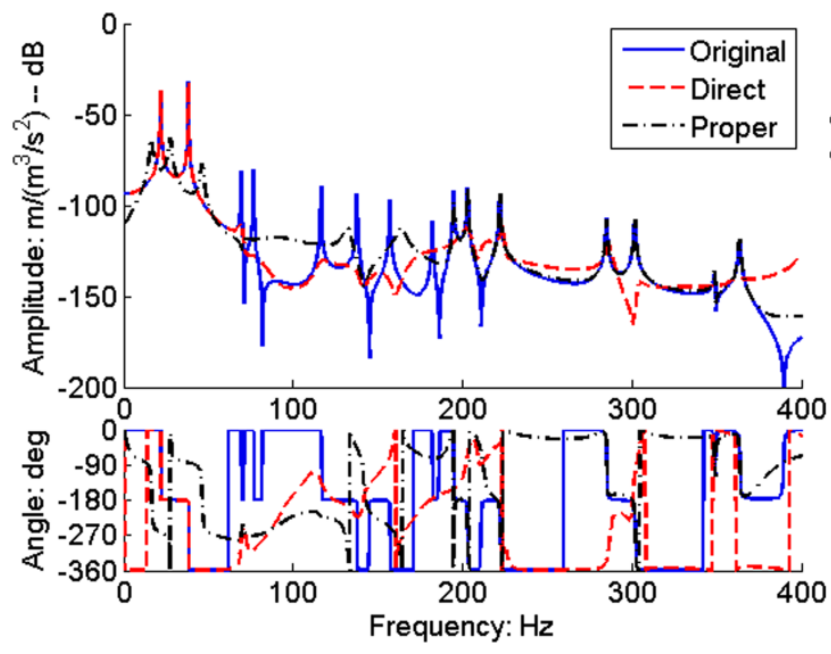

(a) the 5th dof -- structural part

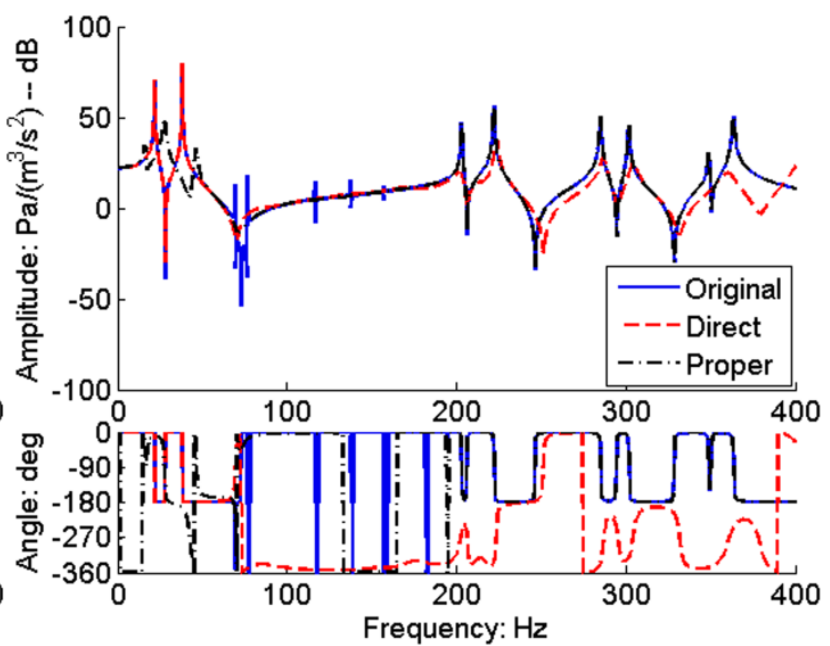

(b) the 15 th dof -- acoustic part

Fig. 6: The original, direct, and proper FRFs of the $5^{\text {th }}$ and $15^{\text {th }}$ dofs for Config-2 


\section{- comparison of the mini-frf FRFs}

As the proper result is not perfect for Config-2, the minimum FRF method is performed as a supplement. This method can be executed on different frequency ranges. As a least-square technique, it clearly has more difficulties on a wider frequency range where more modes need to be fitted. Fig. 7 presents the case when the minimum FRF method is performed on the overall $0-400 \mathrm{~Hz}$ range. Clearly, the optimized FRF fails to fit most of the modes.

An alternative procedure is to divide the overall frequency range into different sub-ranges. The FRF is then corrected separately on the sub-ranges. As the proper FRFs have already fitted with the original data well on 200-400 Hz, only the range of $0-200 \mathrm{~Hz}$ is considered. This range is further divided into four sub-ranges as: 0-50 Hz, 50-100 Hz, 100-150 Hz, and 150-200 Hz. The mini-frf results based on these four sub-ranges are respectively termed as "mini-frf 1-4". Comparison of these corrected FRFs is illustrated in Fig. 8 where interesting results are presented. Taken mini-frf-1 in Fig. 8(a) as an example, the mini-frf-1 FRF fits with the original FRF only on its corresponding sub-range $(0-50 \mathrm{~Hz})$, while on other sub-ranges, the mini-frf-1 FRF is far from the original FRF. Similar results are obtained on the other three cases, implying the minimum FRF method is feasible under specific conditions. An interesting perspective to this approach consists in the determination of frequency-dependent matrices from experimental measurements.

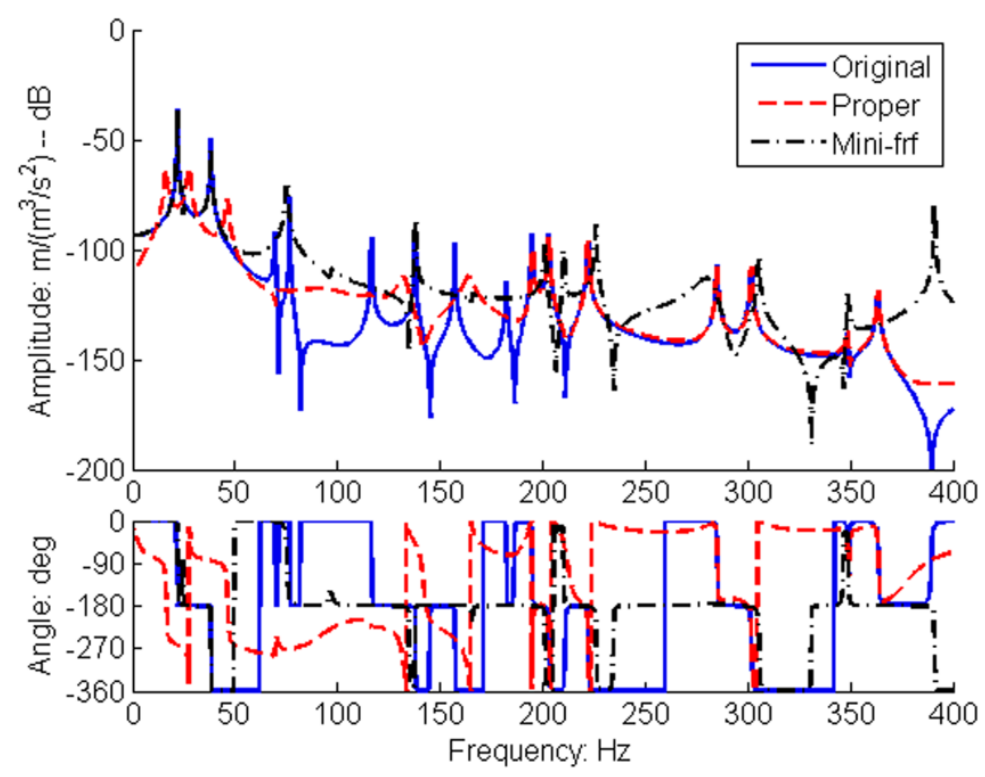

Fig. 7: The original, direct, and mini-frf FRFs on the $5^{\text {th }}$ dof for Config-2

\section{- the natural frequencies corrected by these two methods}

The original and proper natural frequencies for Config-1 and Config-2 are compared in Table 3. Corresponding to the comparison in Figs. 5 and 6, the proper frequencies for Config-1 fit very well with the original data. For Config-2, however, several frequencies inside the range of 0-200 Hz exhibit significant error. 

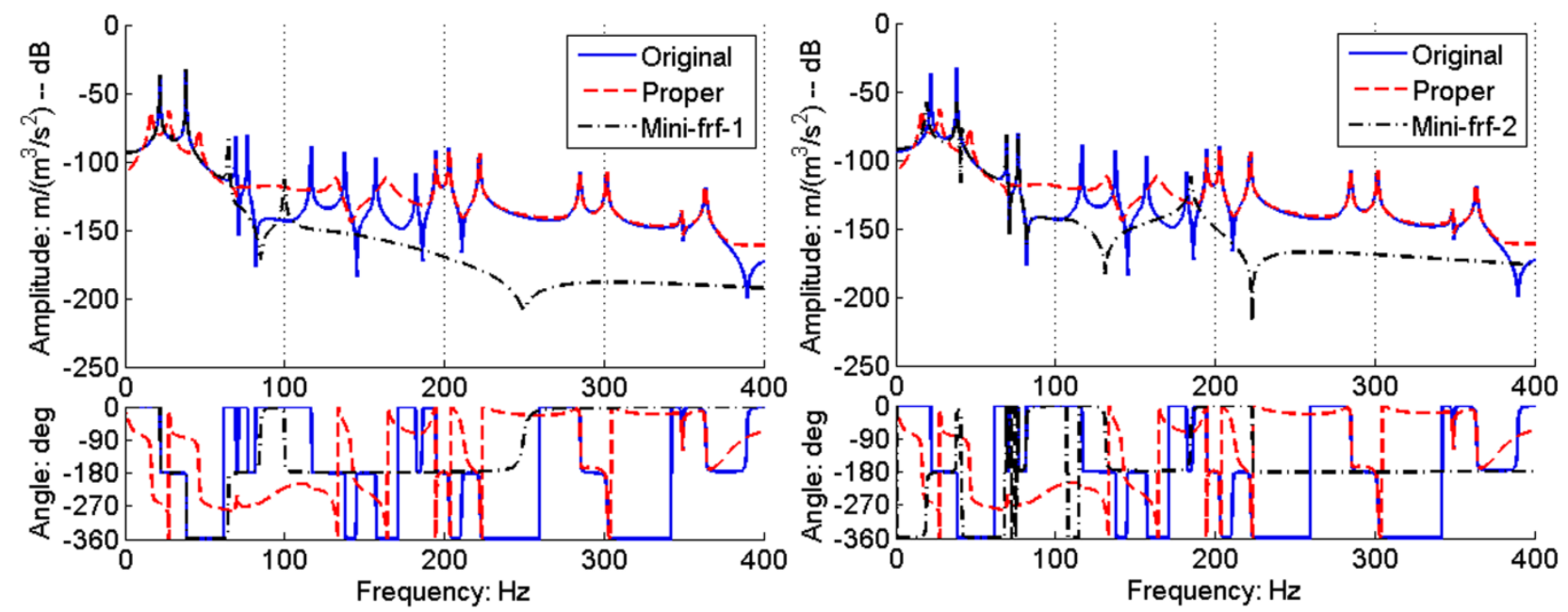

(a) Mini-frf-1 calibrated in sub-range 0-50 Hz;

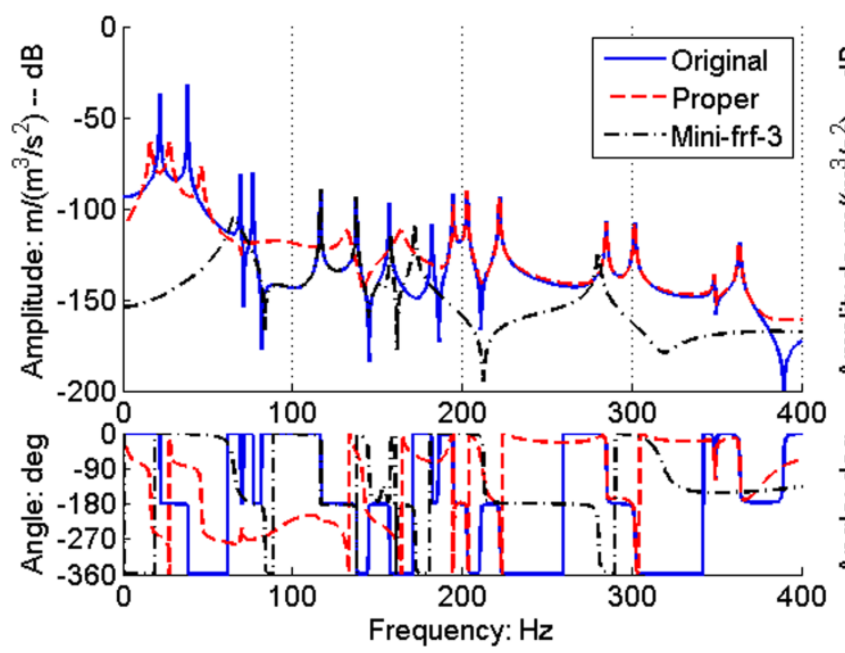

(c) Mini-frf-3 calibrated in sub-range 100-150 Hz;

(b) Mini-frf-2 calibrated in sub-range $50-100 \mathrm{~Hz}$;

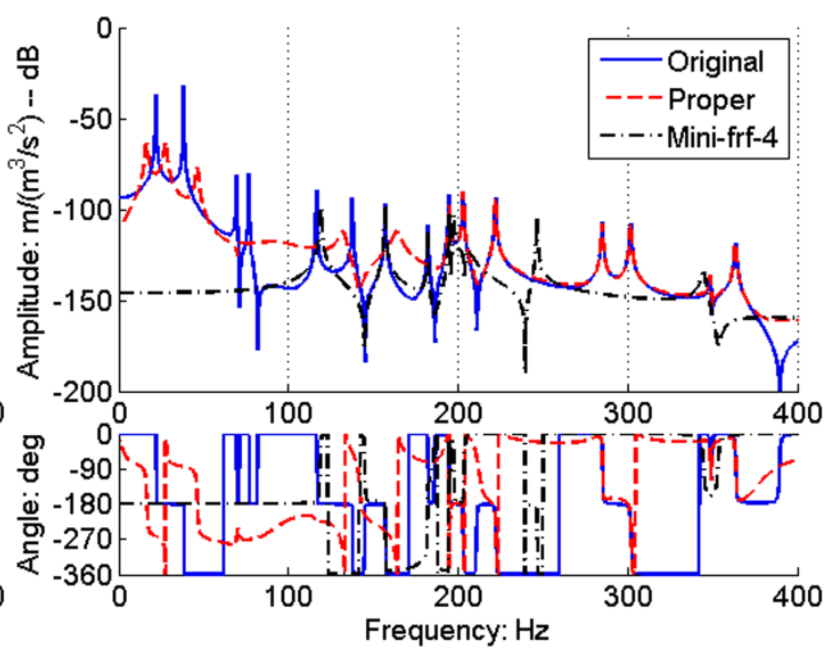

(d) Mini-frf-4 calibrated in sub-range 150-200 Hz;

Fig. 8: Comparison of the mini-frf results on different sub-ranges (performed on the $5^{\text {th }}$ dof for Config-2)

As a supplement, the mini-frf frequencies for Config-2 are listed in Table 4. Recalling Fig. 8, the mini-frf data is only valid on their corresponding sub-ranges. For example, only five modes are presented in mini-frf-1 column, as they lie within the sub-range of $0-50 \mathrm{~Hz}$. By comparing these five frequencies with the original frequency list, it is easy to extract the $3^{\text {rd }}$ and $5^{\text {th }}$ modes as the physical modes which have the same value as the original ones. Other frequencies with strikethrough in this column are ignored. Similarly, physical modes are extracted in the last three columns in Table 4, and the total number of the extracted modes is nine which is the same as the original column.

\subsubsection{Correlation of the decoupled data}

The above comparisons of the FRFs and natural frequencies are about the coupled data, while the decoupled data is also significant for vibroacoustical design. The decoupled data is evaluated by the correlation framework, and the question "which one of Configs 1-4 is the physical configuration?" is answered. For clarity, only the acoustic data is considered in the following context, as the structural data can be evaluated by the same approach. 
Table 3: The original and proper coupled frequencies for Config-1 and Config-2 (\% errors in parentheses)

\begin{tabular}{clllll}
\hline \multirow{2}{*}{$\begin{array}{c}\text { Mode } \\
\text { No. }\end{array}$} & Original & \multicolumn{3}{c}{ Proper frequencies $(\mathbf{H z})$} \\
\cline { 2 - 6 } frequencies $(\mathbf{H z})$ & \multicolumn{2}{c}{ Config-1 } & \multicolumn{2}{c}{ Config-2 } \\
\hline $\mathbf{1}$ & 21.79 & 21.78 & $(-0.1)$ & 21.78 & $(-0.1)$ \\
$\mathbf{2}$ & 38.16 & 38.15 & $(-0.0)$ & 27.24 & $(-28.6)$ \\
$\mathbf{3}$ & 69.35 & 69.29 & $(-0.1)$ & 38.16 & $(-45.0)$ \\
$\mathbf{4}$ & 76.64 & 76.38 & $(-0.3)$ & 71.16 & $(-7.2)$ \\
$\mathbf{5}$ & 116.42 & 117.14 & $(0.6)$ & 85.29 & $(-26.7)$ \\
$\mathbf{6}$ & 137.65 & 137.67 & $(0.0)$ & 116.29 & $(-15.5)$ \\
$\mathbf{7}$ & 157.07 & 157.06 & $(-0.0)$ & 136.38 & $(-13.2)$ \\
$\mathbf{8}$ & 182.20 & 181.85 & $(-0.2)$ & 157.39 & $(-13.6)$ \\
$\mathbf{9}$ & 194.65 & 194.79 & $(0.1)$ & 194.62 & $(-0.0)$ \\
$\mathbf{1 0}$ & 202.98 & 202.98 & $(0)$ & 202.98 & $(0)$ \\
$\mathbf{1 1}$ & 222.03 & 222.03 & $(0)$ & 222.03 & $(0)$ \\
$\mathbf{1 2}$ & 284.93 & 284.93 & $(0)$ & 284.93 & $(0)$ \\
$\mathbf{1 3}$ & 301.70 & 301.70 & $(0)$ & 301.70 & $(0)$ \\
$\mathbf{1 4}$ & 348.77 & 348.77 & $(0)$ & 348.77 & $(0)$ \\
$\mathbf{1 5}$ & 363.49 & 363.49 & $(0)$ & 363.49 & $(0)$ \\
\hline
\end{tabular}

Table 4: The Original and mini-frf frequencies for Config-2 on different sub-ranges

\begin{tabular}{|c|c|c|c|c|c|}
\hline \multirow{2}{*}{$\begin{array}{c}\text { Mode } \\
\text { No. }\end{array}$} & \multirow{2}{*}{ Original (Hz) } & \multicolumn{4}{|c|}{ Config-2 (Hz) } \\
\hline & & Mini-frf-1 & Mini-frf-2 & Mini-frf-3 & Mini-frf-4 \\
\hline & -- & 12.12 & & & \\
\hline & -- & 12.12 & & & \\
\hline \multirow[t]{2}{*}{1} & 21.79 & 21.79 & & & \\
\hline & -- & 28.74 & & & \\
\hline 2 & 38.16 & 38.16 & & & \\
\hline \multirow[t]{2}{*}{3} & 69.35 & & 69.35 & & \\
\hline & -- & & 72.62 & & \\
\hline 4 & 76.64 & & 76.64 & & \\
\hline 5 & 116.42 & & & 1116.42 & \\
\hline \multirow[t]{2}{*}{6} & 137.65 & & & 137.65 & \\
\hline & - & & & & 156.76 \\
\hline 7 & 157.07 & & & & 157.07 \\
\hline 8 & 182.20 & & & & 182.20 \\
\hline \multirow[t]{2}{*}{9} & 194.65 & & & & 194.65 \\
\hline & -- & & & & 197.33 \\
\hline
\end{tabular}

As detailed in Table 5, acoustic frequencies for Configs 1-4 are presented to compare with the coupled data. If there is a frequency continually appears in different configurations, then this frequency is termed as "steady" frequency. The steady frequencies are marked with bold and italic, and the unsteady frequencies are marked with strikethrough. For clarity, the steady frequencies in different configurations are placed in the same row as their corresponding coupled frequency. All frequencies for Config-3 are steady, and each of these frequencies has a corresponding value in the coupled column. This implies Config-3 is the physical configuration in this 
example. Note that the $1^{\text {st }}$ frequencies for Configs 1-3 have an obvious discrepancy compared with the $1^{\text {st }}$ coupled frequency. This is because the $1^{\text {st }}$ acoustic mode has a strong coupling relationship with the first two coupled modes, as it will be shown later.

Table 5: Correlation of the decoupled acoustic frequencies for different configurations

\begin{tabular}{cccccc}
\hline \multirow{2}{*}{ Mode No. } & Coupled & \multicolumn{4}{c}{ Decoupled acoustic (Hz) } \\
\cline { 3 - 6 } & $(\mathrm{Hz})$ & Config-1 & Config-2 & Config-3 & Config-4 \\
\hline 1 & 21.79 & 26.78 & 28.33 & 28.34 & 37.31 \\
2 & 38.16 & 47.41 & 99.26 & & 218.81 \\
3 & 69.35 & 125.10 & & & 233.47 \\
4 & 76.64 & & & & 300.01 \\
5 & 116.42 & & & & 323.71 \\
6 & 137.65 & & & & 360.66 \\
7 & 157.07 & & & & \\
8 & 182.20 & & 202.96 & 202.95 & \\
9 & 194.65 & & 222.03 & 221.33 & \\
10 & 202.98 & 202.97 & 284.28 & 282.71 & \\
11 & 222.03 & 222.03 & 301.66 & 301.30 & \\
12 & 284.93 & 284.82 & 348.82 & 348.12 & \\
13 & 301.70 & 301.96 & 363.49 & 363.38 & \\
14 & 348.77 & 348.93 & & & \\
15 & 363.49 & 363.49 & & & \\
\end{tabular}

The above conclusion is also demonstrated by the MAC matrices between the acoustic eigenvector and the coupled eigenvector as shown in Fig. 9. For different configurations, the MAC matrices clearly have different sizes, and they are respectively termed as "MAC-Configs 1-4". Taken Fig. 9(b) as an example, the size of MAC-Config- 2 is 8 -by- 15 . The 8 rows denote the 8 decoupled acoustic modes, and the 15 columns denote the 15 coupled modes. The idea is that when there is a row where only one element is close to one and others are close to zero, then this row denotes a physical decoupled mode. And its corresponding coupled mode is also decided by the column number of the element closing to one. Under this sense, the $4^{\text {th }}-8^{\text {th }}$ modes are physical and their corresponding coupled modes are the $11^{\text {th }}-15^{\text {th }}$ modes in the coupled column. The $1^{\text {st }}$ and $3^{\text {rd }}$ rows do not have the exactly same feature as the $4^{\text {th }}-8^{\text {th }}$ rows, but rows with the similar style can be found in MAC-Config-1 and MAC-Config-3, implying these two modes are also steady. The $2^{\text {nd }}$ row in MAC-Config-2 has 6 elements close to one, and no similar row can be found in other MACs. Consequently, the $2^{\text {nd }}$ acoustic mode of Config-2 is a redundant mode. Finally, the number of physical acoustic modes is determined as 7. The $1^{\text {st }}$ acoustic mode is corresponding to the $1^{\text {st }}$ coupled mode, and the other acoustic modes are corresponding to the $10^{\text {th }}-15^{\text {th }}$ coupled modes. This correlation result is same as the conclusion obtained from the natural frequencies. 


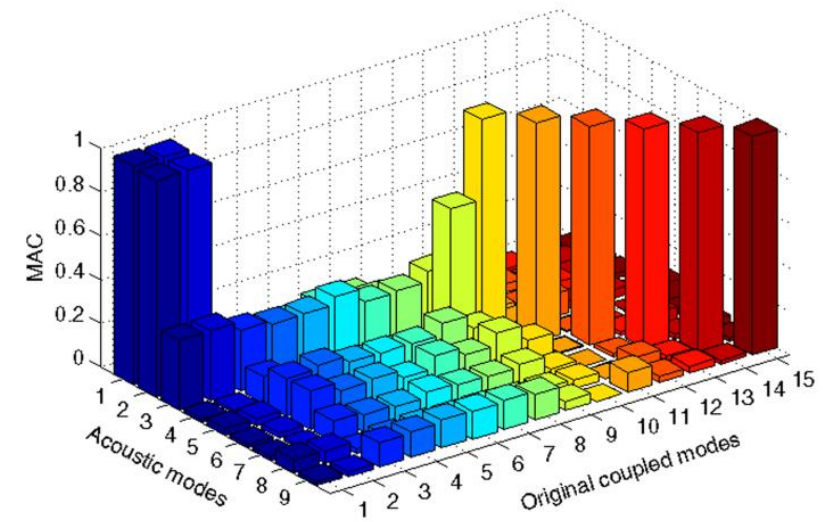

(a) MAC-Config-1

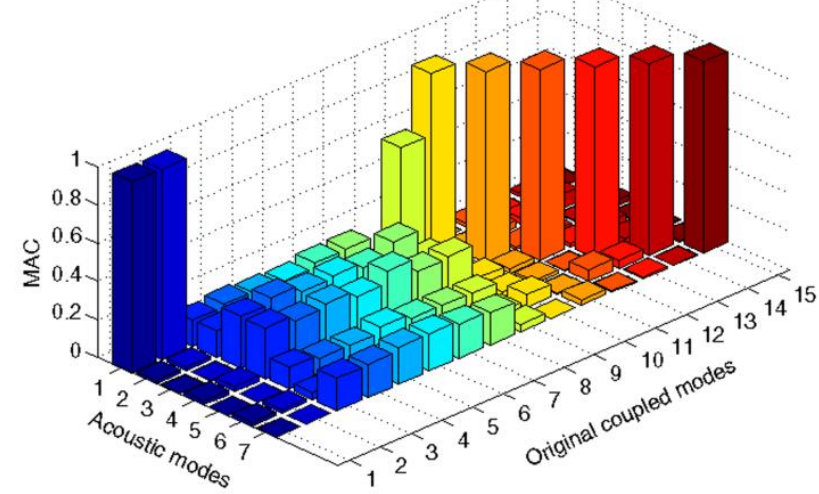

(c) MAC-Config-3

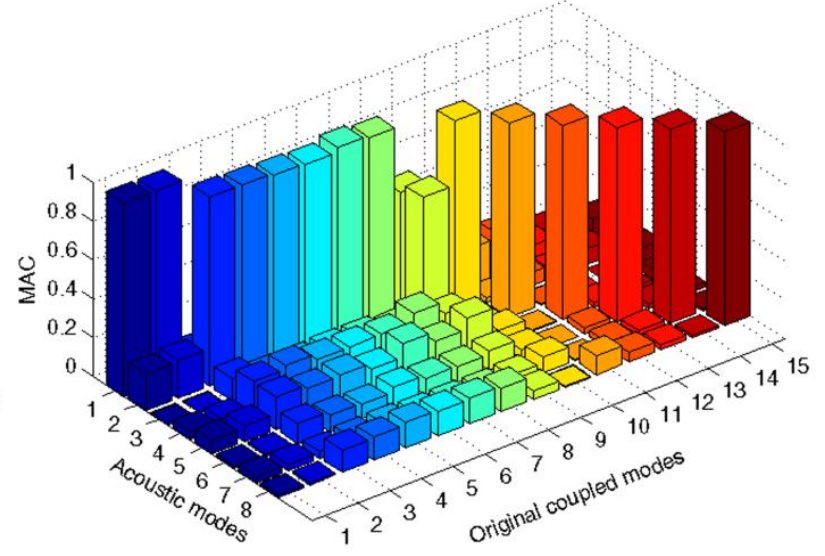

(b) MAC-Config-2

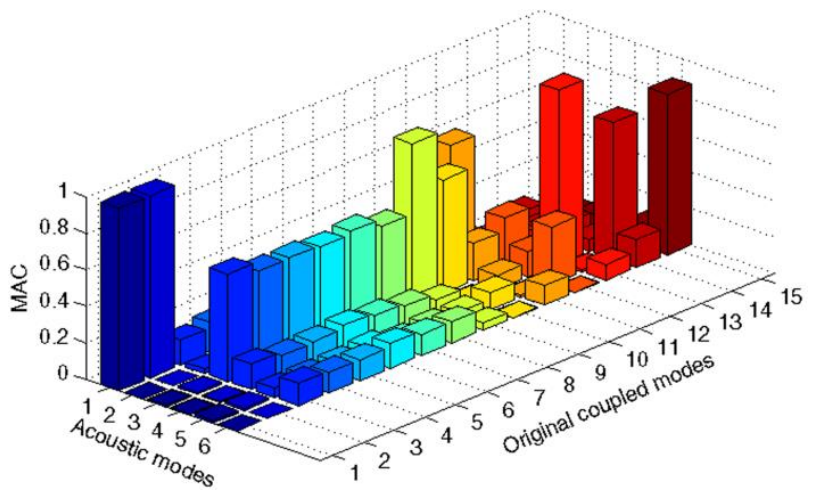

(d) MAC-Config-4

Fig. 9: MAC matrices between the acoustic and coupled eigenvectors for different configurations

More attention should be taken on the $1^{\text {st }}$ and $2^{\text {nd }}$ acoustic modes in Fig. 9 (c). There are two elements close to one in the $1^{\text {st }}$ row, and the $9^{\text {th }}$ element in the $2^{\text {nd }}$ row is not minimized to zero. These strange features can be explained by the strong coupling between these modes. This can be checked in Table 1 where the $1^{\text {st }}$ acoustic frequency has a value in the middle of the $1^{\text {st }}$ and the $2^{\text {nd }}$ coupled frequency. In other words, the strong coupling makes the $1^{\text {st }}$ acoustic mode dependent with more than one mode in the coupled mode list.

Compared with the first three configurations, Config-4 provides chaotic acoustic modes where neither the natural frequencies nor the modal shapes correspond to the coupled modes. This means that when the proposed dofs number is greater than the actual number, this kind of configuration can provide physical modes plus some redundant modes. But when the proposed dofs number is lower than the actual one, most of the obtained modes are unphysical. Hence Config- 4 is not utilized in the correlation procedure.

Based on the above analysis, key principles of correlation are summarized:

i. if a mode is steady, and its corresponding row in MAC has only one element close to one and others are minimized to zero, this mode is a physical mode with weak coupling to other modes;

ii. if a mode is steady, and its corresponding row in MAC has more than one element close to one, this mode is a physical mode with strong coupling to other modes;

iii. if a mode is unsteady in different configurations, this mode is a redundant mode because the proposed dofs in this configuration are more than the actual number;

iv. if all modes are unsteady in a configuration, the proposed dofs are less than the actual number of modes.

These principles are important for practical engineering where more noise and stronger coupling 
relationships are present in the experimental measurement.

\subsubsection{Summary}

The illustration on this 253 dofs simulated case study is detailed and it involves most of the issues which the engineer should encounter in practical application. Key steps and their outcomes are summarized to generate a clear understanding of this integrated approach.

1. 15 coupled modes are identified in the frequency range of interest $(0-400 \mathrm{~Hz})$;

2. Size of the modes is reduced from 253 to 15 , with the consideration of different configurations (e.g. $6+9,7+8, \ldots)$;

3. The reduced modes are firstly optimized by the properness enforcement method, and the minimum FRF method is employed as a supplement on some specific frequency ranges where the proper data is not perfect enough.

4. Based on the correlation framework, Config-3 (i.e. $8+7$ ) is determined as the physical configuration;

5. As a final outcome, the reduced and decoupled system matrices with the physical configuration are obtained, and the predicted FRF, natural frequencies, and eigenvectors fit with the original data well.

\subsection{The experimental case study}

\subsubsection{Description of the experimental setup}

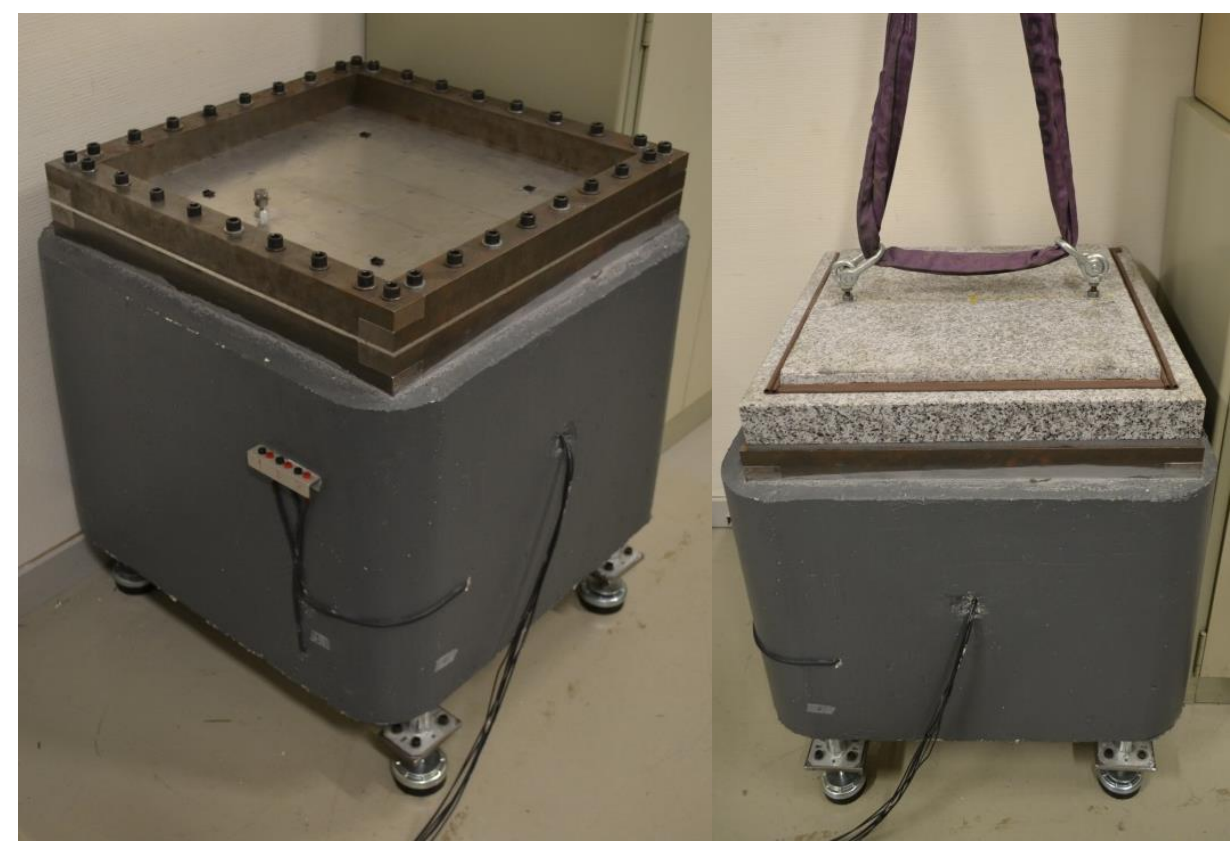

(a) with the aluminum plate

(b) with the marble cover

Fig. 10: The experimental setup with different cover of the cavity.

The experiment is conducted on a cavity surrounded by concrete and closed in the top surface with a $5 \mathrm{~mm}$ thickness aluminum plate, as shown in Fig. 10(a). There are 5 accelerometers on the plate and 6 microphones inside the cavity, indicating that the measured FRF has 11 components, corresponding to a maximum of 5 structural dofs and 6 acoustic dofs in the reduced model. The excitation is applied by a loudspeaker inside the cavity. The frequency range considered herein is $300-500 \mathrm{~Hz}$.

In this real case, the environment noise and uncertainties in the experimental setup play important role in the identification and have a significant influence on the final outcome. This influence can be first checked in Fig. 11 where two sets of measured FRFs are respectively identified. The upper and lower FRFs are separately 
measured with different sealing effectiveness, sensor sensitivities, and sensor placements. Ideally when there is no noise and the setup is perfectly constructed, these two sets should have the same identification result. However, the fact is some modes obviously shift. The modes which are steady in both cases are selected as shown in Fig. 11. This strategy leads to loss of modes, but as described in Section 5.1.1, loss of modes is unavoidable even in a simulated case. On the other hand, this strategy can make sure every identified mode is physical.

Six modes are identified as listed in Table 6. The identified frequencies and their corresponding FRF are used as reference data. Besides these coupled data, the decoupled reference data is also provided. In this example, the decoupled data for the cavity is measured by replacing the aluminum plate by a marble cover as shown in Fig. 10(b). The surrounding concrete and marble cover are approximately regarded as rigid, so that the measured data is served as reference of the decoupled acoustic sub-model.
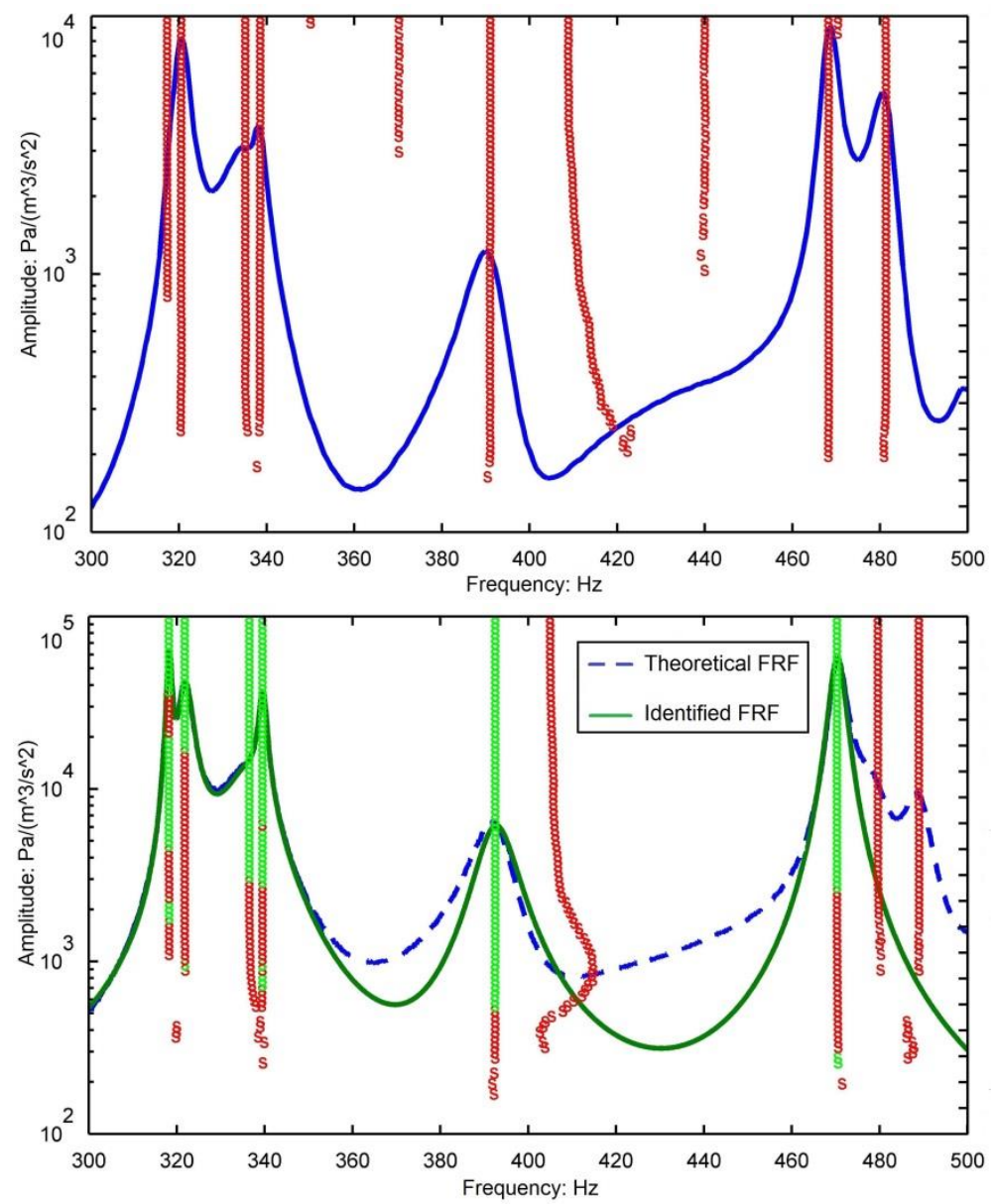

Fig. 11: Selection of the poles in identification.

\subsubsection{Reduction and optimization}

Reduction is performed on the identified eigenvectors to reduce their dofs from 11 to 6 . The four configurations considered herein $(1+5,2+4,3+3$, and $4+2)$ are respectively termed as "Configs $1-4$ ". First, the reduced modes are optimized by the properness enforcement method. The proper natural frequencies (Table 6) and FRFs (Fig. 12) are respectively compared with the identified and direct data. For clarity, only the results for Config-1 and Config-2 are presented. The direct natural frequencies exhibit obvious errors compared with the identified ones, while the proper frequencies have smaller errors for Config- 1 or Config- 2 , as shown in Table 6. 
Table 6: The identified, direct and proper frequencies for different configurations (\% errors in parentheses)

\begin{tabular}{|c|c|c|c|c|c|c|c|}
\hline \multirow{3}{*}{$\begin{array}{l}\text { Mode No. } \\
1\end{array}$} & \multirow{3}{*}{$\begin{array}{l}\text { Identified (Hz) } \\
318.3\end{array}$} & \multirow{2}{*}{\multicolumn{2}{|c|}{ Direct (Hz) }} & \multicolumn{4}{|c|}{ Proper (Hz) } \\
\hline & & & & \multicolumn{2}{|c|}{ Config-1 } & \multicolumn{2}{|c|}{ Config-2 } \\
\hline & & 326.3 & $(2.5)$ & 317.4 & $(-0.3)$ & 317.8 & $(-0.2)$ \\
\hline 2 & 321.8 & 339.5 & (5.5) & 332.8 & (3.4) & 325.7 & $(1.2)$ \\
\hline 3 & 336.6 & 346.9 & (3.1) & 339.1 & $(0.7)$ & 336.9 & $(0.1)$ \\
\hline 4 & 339.6 & 386.0 & $(13.7)$ & 354.1 & $(4.3)$ & 349.5 & $(2.9)$ \\
\hline 5 & 392.6 & 386.0 & $(-1.6)$ & 370.1 & $(-5.7)$ & 380.1 & $(-3.2)$ \\
\hline 6 & 470.4 & 422.4 & $(-10.2)$ & 460.9 & $(-2.0)$ & 466.6 & $(-0.8)$ \\
\hline \multicolumn{2}{|c|}{ Mean absolute error } & $6.1 \%$ & & $2.7 \%$ & & $1.4 \%$ & \\
\hline
\end{tabular}

Because of the noise and damping in the measurement, Fig. 12 shows the direct FRFs completely fail in representing the identified FRFs, while the proper FRFs are obviously improved compared with the direct ones. The proper FRFs for Config- 1 and Config -2 are similar with each other, and they fit with the identified FRF on the whole frequency range except on the sub-range nearby $400 \mathrm{~Hz}$. This discrepancy is more obvious for the structural dofs as shown in Fig. 12(a). The possible explanation comes from the imperfect measurements. The mode nearby $400 \mathrm{~Hz}$ corresponds to the acoustic part making the response on the plate much smaller than other structural resonance modes. As a result, the involved accelerometers may be not sensitive enough to measure the response on the plate.

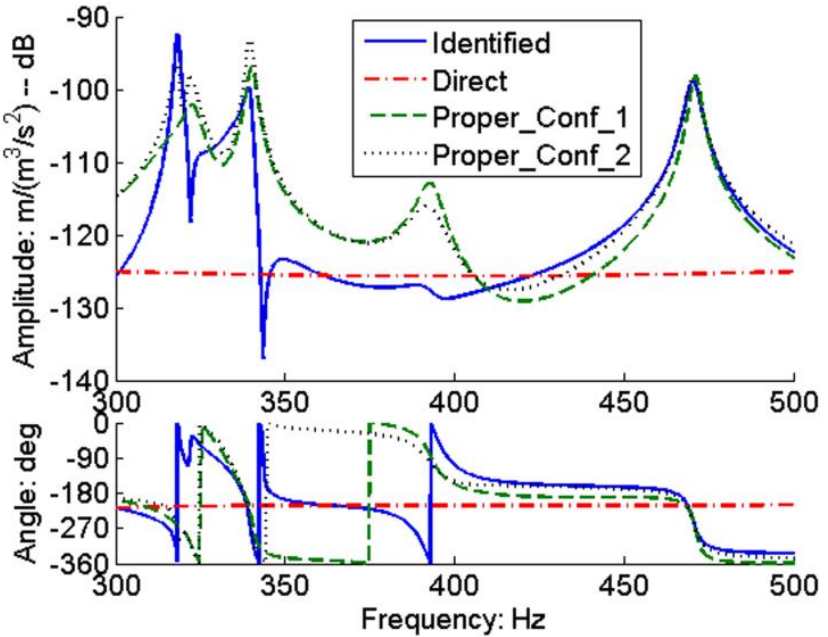

(a) the $1^{\text {st }}$ dof -- structural part

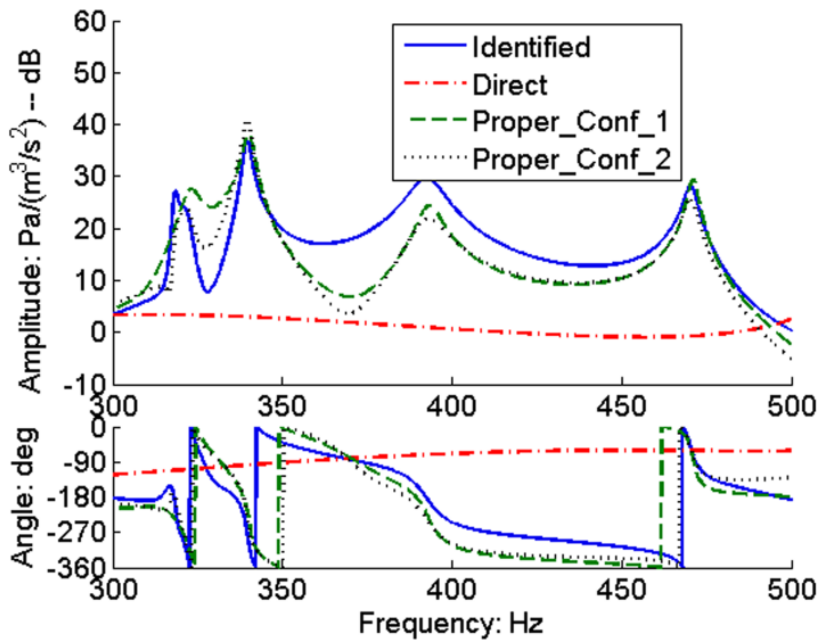

(b) the $6^{\text {th }}$ dof -- acoustic part

Fig. 12: The identification, direct, and proper FRFs for Configs 1 and 2

As shown in Fig. 11, four modes are identified on the sub-range of 300-360 Hz. These closely spaced modes in such a narrow frequency range lead to obvious difficulties for decoupling identification. The minimum FRF method is consequently evaluated on this specific part. It is performed separately on the sub-ranges of $300-330 \mathrm{~Hz}$ and $320-360 \mathrm{~Hz}$, with the results respectively labeled as "mini-frf-1" and "mini-frf-2". The identified, proper, and mini-frf FRFs are presented in Fig. 13. Similar as the simulated example (Fig. 8), the mini-frf FRFs fit with the identified FRFs in their corresponding sub-ranges. 


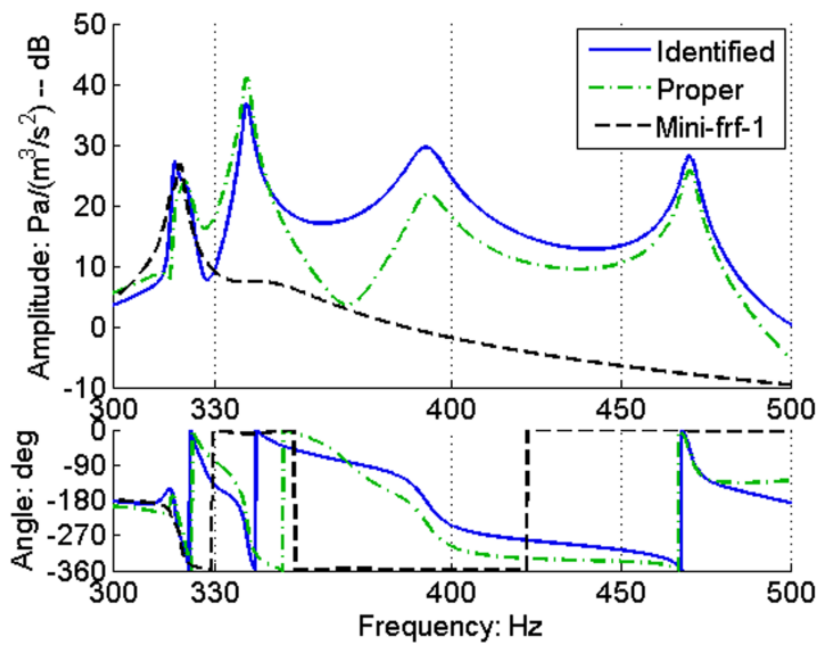

(a) Mini-frf-1 calibrated in sub-range 300-330 Hz;

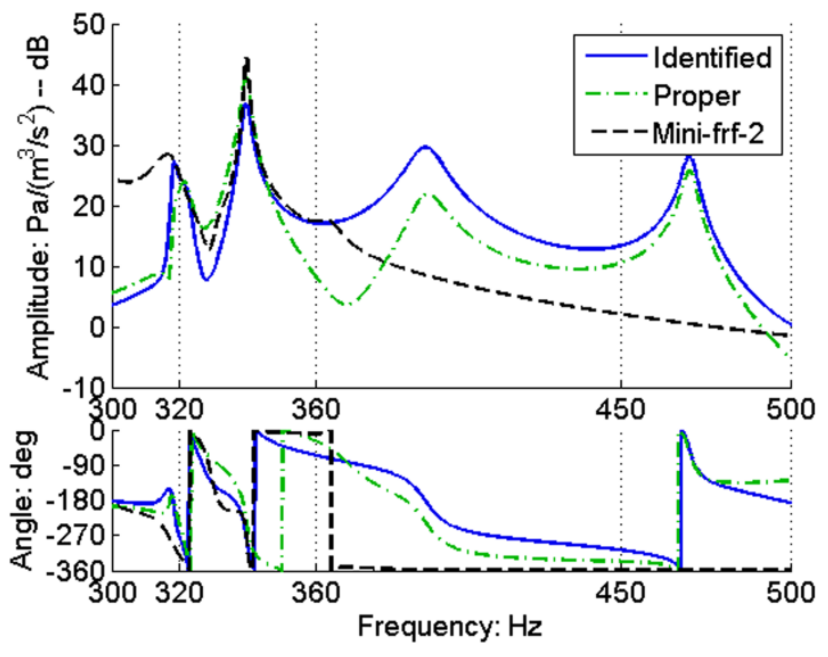

(b) Mini-frf-2 calibrated in sub-range $320-360 \mathrm{~Hz}$;

Fig. 13: mini-frf optimized FRFs of the $6^{\text {th }}$ dof (acoustic) for Config-2

The natural frequencies within the considered range of $300-360 \mathrm{~Hz}$ are listed in Table 7. Two frequencies are reserved in the column of mini-frf-1, as they lie in the sub-range of 300-330 Hz. Similarly three frequencies are reserved in the column of mini-frf-2. It is interesting to note that the same frequency value (320.6 Hz) appears in both columns. This is because the two sub-ranges have an overlapping range as 320-330 Hz. The last column of Table 7 is the combined frequencies extracted from the columns of mim-frf- 1 and mini-frf-2. The mean absolute error compared with the identified data is $1.00 \%$ which is relatively smaller than the proper data (Table 6).

Table 7: The identified and mini-frf frequencies within the range of 300-360 Hz for Config-2 (\% errors in parentheses)

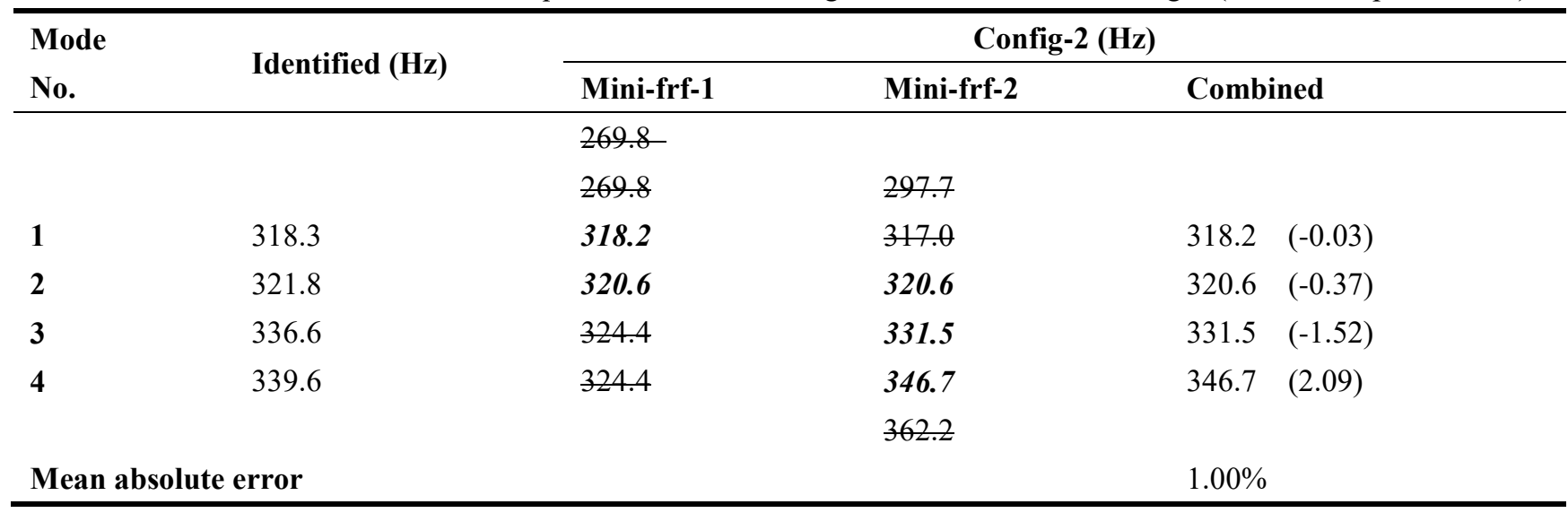

\subsubsection{Correlation of the decoupled model}

The coupled frequencies and the decoupled acoustic frequencies for each configuration are listed in Table 8 . For this experimental case, the correlation is more difficult than the one which has been performed in the simulated case because of the noise and coupling relationships between the modes. It is not easy to recognize a steady value in different configurations especially for the closely spaced frequencies. However, it is still possible to recognize that the last two frequencies (bold and italic) are steady in both Config-1 and Config-2, and they correspond to the last two values in the coupled column as shown in Table 8. This statement is confirmed with the eigenvector correlation. The MAC matrices between acoustic eigenvectors and the coupled eigenvectors under Configs 1-4 (termed as "MAC 1-4") are presented in Fig. 14. Following the principles extracted in Section 5.1.3, the correlation results are summarized as below.

1. Based on Principle (i), the last two rows in MAC-1 and MAC-2 are steady and only one element in each 
row close to one. So the last two modes for Config-1 and Config-2 are physical modes with weak coupling, and they correspond to the last two coupled modes.

2. The $2^{\text {nd }}$ row in MAC- 1 has the similar style as the $1^{\text {st }}$ row in MAC-2; the $3^{\text {rd }}$ row in MAC- 1 has the similar style as the $2^{\text {nd }}$ row in MAC-2. Based on Principle (ii), the $2^{\text {nd }}$ and $3^{\text {rd }}$ modes for Config- 1 are physical modes with strong coupling, and they correspond to the first two coupled modes.

3. The $1^{\text {st }}$ row in MAC-1 has no similar row in other MACs. Based on Principle (iii), the $1^{\text {st }}$ mode for Config-1 is a redundant mode.

4. All modes for Config-3 and Config-4 are unsteady. Based on Principle (iv), the proposed number of dofs is lower than the actual number. Config-2 is demonstrated as the physical configuration (i.e. $2+4$ ) for this experimental case. In other words, among the six coupled modes, the $1^{\text {st }}, 2^{\text {nd }}, 5^{\text {th }}$, and $6^{\text {th }}$ modes correspond to the cavity; while the $3^{\text {rd }}$ and $4^{\text {th }}$ modes correspond to the plate.

Table 8: Correlation between the coupled frequencies and the acoustic frequencies for different configurations

\begin{tabular}{clcccc}
\hline \multirow{2}{*}{ Mode No. } & \multirow{2}{*}{ Coupled (Hz) } & \multicolumn{5}{c}{ Acoustic (Hz) } \\
\cline { 3 - 6 } & -- & Config-1 & Config-2 & Config-3 & Config-4 \\
\hline $\mathbf{1}$ & 318.3 & 328.3 & & 320.0 & 269.0 \\
$\mathbf{2}$ & 321.8 & $\mathbf{3 3 5 . 4}$ & $\mathbf{3 3 4 . 7}$ & 345.2 & 348.0 \\
$\mathbf{3}$ & 336.6 & $\mathbf{3 5 1 . 1}$ & $\mathbf{3 4 2 . 2}$ & 441.9 & \\
$\mathbf{4}$ & 339.6 & & & & \\
$\mathbf{5}$ & 392.6 & & & & \\
$\mathbf{6}$ & 470.4 & $\mathbf{3 8 4 . 5}$ & $\mathbf{3 9 1 . 7}$ & \\
\hline
\end{tabular}

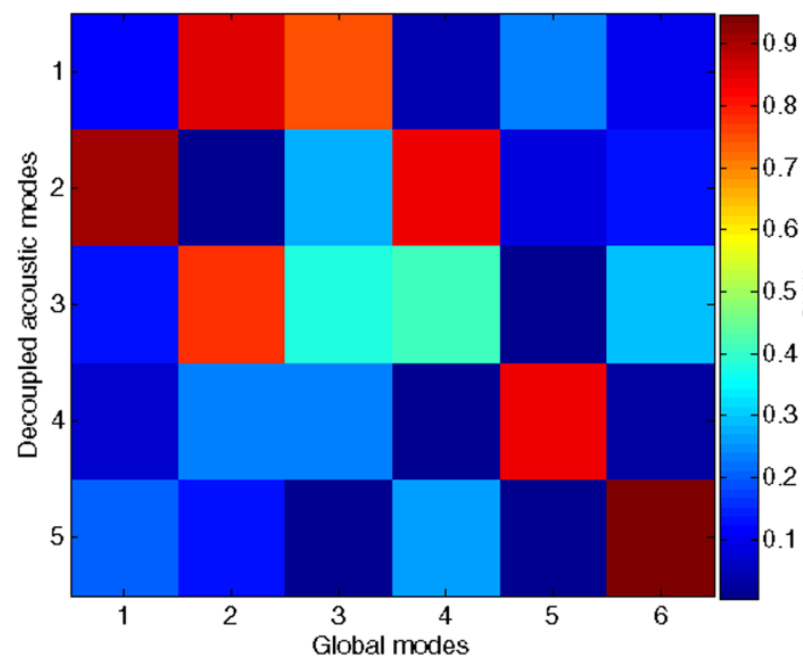

(a) MAC-1

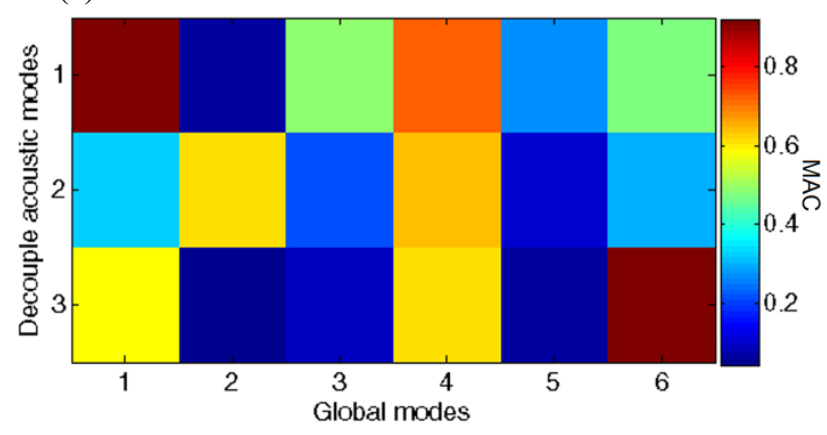

(c) MAC-3

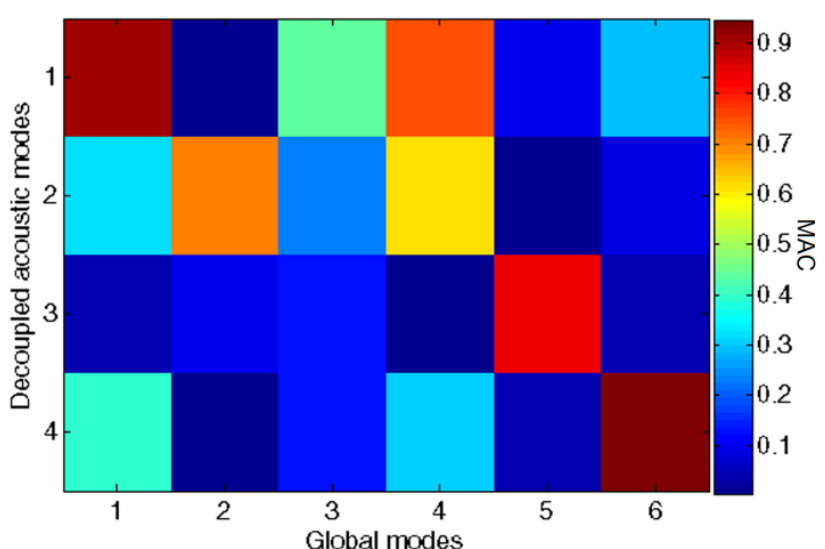

(b) $\mathrm{MAC}-2$

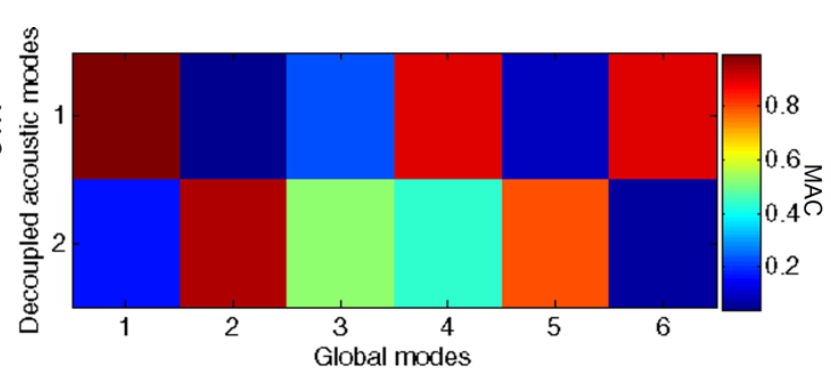

(d) MAC-4

Fig. 14: MAC matrices between the coupled modes and the acoustic modes with different configurations 
The finally identified acoustic sub-model is evaluated according to the experimental reference data measured as Fig. 10(b). Config-2 is confirmed again as the physical configuration in Table 9 where four modes are experimentally measured in the range of 300-500 Hz. The mean absolute error of the predicted frequencies is only $1.38 \%$, showing the identified model can reproduce the same behavior as the measured one.

Table 9: Acoustic frequencies from the experiment and the identified model for Config-2 (\% errors in parentheses)

\begin{tabular}{lllllc}
\hline Mode No. & $\mathbf{1}$ & $\mathbf{2}$ & $\mathbf{3}$ & $\mathbf{4}$ & Mean absolute error \\
\hline Experimental (Hz) & 328.8 & 334.9 & 387.9 & 475.9 & \\
Config-2 (Hz) & $334.7(1.8 \%)$ & $342.2(2.2 \%)$ & $391.7(1.0 \%)$ & $473.2(-0.6 \%)$ & $1.38 \%$ \\
\hline
\end{tabular}

\section{Conclusions and prospects}

This work concentrates on a thorough guidance for vibroacoustical identification with the consideration of various obstacles encountered in real application. In particular, a methodology allowing the decoupling of structural and acoustic effects from coupled measurements has been proposed. Compared with the authors' previous work on this field, further contributions are summarized as follows:

1. The correlation procedure is capable of filtering the redundant decoupled modes, so that the structural/acoustic sub-model can represent the system behavior;

2. The integrated application of minimum FRF method and properness enforcement method provides satisfied outcome even in complex cases with high level noise and closed modes;

3. The extended LSCF method is specially developed for vibroacoustics where the right and left eigenvectors are separately identified;

4. The QR reduction technique significantly reduced the dofs of the identified model, ensuring the overall approach's feasibility in practical application.

Two meaningful extensions of this proposed approach are expected: First, a deeper insight into the damping terms. A physical description of damping in vibroacoustics is significant to be further evaluated. Second, a higher frequency application involving more challenging features (e.g. nonnegligible uncertainty and stronger coupling) is necessary to be deeply studied in the next step. Furthermore, the experimental case clearly shows that the measurement noise exhibits significant influence on the overall approach. A sophisticated experimental setup along with the MIMO identification strategy should bring better outcomes.

\section{Acknowledgement}

The Authors gratefully acknowledge Paul Lépine for his contributions on the measurements utilized in the experimental case study in this paper. This work was co-financed by the French National Research Agency under grant number ANR-12-JS09-008-COVIA. It has been performed in cooperation with the Labex ACTION program (ANR-11-LABX-0001-01). The authors wish to thank Marianne Sagnard, Barbara Roux, Anthony Boillot, Alexis Rogeon and Otmane Zemzem, the students who have contributed to this work, and Bruno Chamont, the low and mid frequency team manager of PSA Group, for providing financial support to this project. 


\section{Reference}

[1] H. Tsuji, S. Maruyama, T. Yoshimura, E. Takahashi, Experimental method extracting dominant acoustic mode shapes for automotive interior acoustic field coupled with the body structure, SAE International Journal of Passenger Cars-Mechanical Systems, 6 (2013) 1139-1146.

[2] P. Gardonio, Review of active techniques for aerospace vibro-acoustic control, Journal of aircraft, 39 (2002) 206-214.

[3] S. Dhandole, S. Modak, Review of vibro-acoustics analysis procedures for prediction of low frequency noise inside a cavity, Proceedings of the Conference \& Exposition on Structural Dynamics, Orlando, FL, USA, (2007) 19-22.

[4] S. Ibrahim, A. Sestieri, Existence and normalization of complex modes in post experimental use in modal analysis, Proceedings of the 13th International Modal Analysis Conference, (1995) 483-489.

[5] P. Lancaster, U. Prells, Inverse problems for damped vibrating systems, Journal of Sound and Vibration, 283 (2005) 891-914.

[6] Q. Zhang, G. LALLEMENT, Comparison of normal eigenmodes calculation methods based on identified complex eigenmodes, Journal of Spacecraft and Rockets, 24 (1987) 69-73.

[7] E. Balmés, New results on the identification of normal modes from experimental complex modes, Mechanical Systems and Signal Processing, 11 (1997) 229-243.

[8] M. Ouisse, E. Foltête, On the properness condition for modal analysis of non-symmetric second-order systems, Mechanical Systems and Signal Processing, 25 (2011) 601-620.

[9] M. Ouisse, E. Foltête, Model correlation and identification of experimental reduced models in vibroacoustical modal analysis, Journal of Sound and Vibration, 342 (2015) 200-217.

[10] K. Wyckaert, F. Augusztinovicz, P. Sas, Vibro - acoustical modal analysis: Reciprocity, model symmetry, and model validity, The Journal of the Acoustical Society of America, 100 (1996) 3172-3181.

[11] C.S. Wu, D.L. Young, C.M. Fan, Frequency response analyses in vibroacoustics using the method of fundamental solutions, Computational Mechanics, 47 (2011) 519-533.

[12] J.E. Mottershead, M. Link, M.I. Friswell, The sensitivity method in finite element model updating: A tutorial, Mechanical Systems and Signal Processing, 25 (2011) 2275-2296.

[13] Z. Deng, S. Bi, S. Atamturktur, Stochastic model updating using distance discrimination analysis, Chinese Journal of Aeronautics, 27 (2014) 1188-1198.

[14] S. Bi, Z. Deng, Z. Chen, Stochastic Validation of Structural FE-models Based on Hierarchical Cluster Analysis and Advanced Monte Carlo Simulation, Finite Elements in Analysis and Design, 67 (2013) 22-33.

[15] A. Calvi, Uncertainty-based loads analysis for spacecraft: Finite element model validation and dynamic responses, Computers \& Structures, 83 (2005) 1103-1112.

[16] O. Dazel, F. Sgard, C.-H. Lamarque, N. Atalla, An extension of complex modes for the resolution of finite-element poroelastic problems, Journal of Sound and Vibration, 253 (2002) 421-445.

[17] M. Ouisse, M. Ichchou, S. Chedly, M. Collet, On the sensitivity analysis of porous material models, Journal of Sound and Vibration, 331 (2012) 5292-5308.

[18] F. Wu, G.R. Liu, G.Y. Li, A.G. Cheng, Z.C. He, Z.H. Hu, A novel hybrid FS-FEM/SEA for the analysis of vibro-acoustic problems, International Journal for Numerical Methods in Engineering, 102 (2015) 1815-1829.

[19] M.C. Bampton, J. CRAIG, Roy R, Coupling of substructures for dynamic analyses, AIAA Journal, 6 (1968) 1313-1319.

[20] D.D. Klerk, D.J. Rixen, S. Voormeeren, General framework for dynamic substructuring: history, review and classification of techniques, AIAA Journal, 46 (2008) 1169-1181. 
[21] R.J. Allemang, The modal assurance criterion: twenty years of use and abuse, Sound and vibration, 8 (2003) 14-21.

[22] H. Van Der Auweraer, P. Guillaume, P. Verboven, S. Vanlanduit, Application of a fast-stabilizing frequency domain parameter estimation method, Journal of dynamic systems, measurement, and control, 123 (2001) 651-658.

[23] B. Peeters, H. Van der Auweraer, P. Guillaume, J. Leuridan, The PolyMAX frequency-domain method: a new standard for modal parameter estimation? Shock and Vibration, 11 (2004) 395-409.

[24] C. Schedlinski, M. Link, An approach to optimal pick-up and exciter placement, Proceedings-SPIE the international society for optical engineering, SPIE international society for optical, (1996) 376-382. 